\title{
Extended Analytical Formulas for the Perturbed Keplerian Motion Under a Constant Control Acceleration
}

\author{
Federico Zuiani
}

Ph.D. Candidate, University of Glasgow, Glasgow, United Kingdom.

Massimiliano Vasile

Professor, University of Strathclyde, Glasgow, United Kingdom.

\begin{abstract}
This paper presents a set of analytical formulae for the perturbed Keplerian motion of a spacecraft under the effect of a constant control acceleration. The proposed set of formulae can treat control accelerations that are fixed in either a rotating or inertial reference frame. Moreover, the contribution of the $J_{2}$ zonal harmonic is included in the analytical formulae. It will be shown that the proposed analytical theory allows for the fast computation of long, multi-revolution spirals while maintaining good accuracy. The combined effect of different perturbations and of the shadow regions due to solar eclipse is also included. Furthermore, a simplified control parameterisation is introduced to optimise thrusting patterns with two thrust arcs and two cost arcs per revolution. This simple parameterisation is shown to ensure enough flexibility to describe complex low thrust spirals. The accuracy and speed of the proposed analytical formulae are compared against a full numerical integration with different integration schemes. An averaging technique is then proposed as an application of the analytical formulae. Finally, the paper presents an example of design of an optimal low-thrust spiral to transfer a spacecraft from an elliptical to a circular orbit around the Earth.
\end{abstract}

Keywords: low-thrust trajectories, analytical solutions, first order expansions

\section{INTRODUCTION}

Analytical solutions have been proposed for the integration of long, low thrust, multirevolution transfers under the assumption of small eccentricity (Kechichian 1997a, 1997b, 1998a, 1998b, 1998c, 2007; Casalino and Colasurdo 2007), and averaging techniques (Geffroy and Epenoy 1997) (Kluever and Oleson 1998; Gao and Li 1998) exist for designing generic many-revolution transfers both with indirect and direct approaches. In particular, the works of J.A. Kechichian proposed various analytical or semi-analytical solutions to specific trajectory design problems. Kechichian 1998a tackled the problem of the planar, eccentricityconstrained, low-thrust orbit raising, producing a closed form solution for the thrust control which takes advantage of the condition of constant eccentricity. Kechichian 1998b instead dealt with the problem of orbit raising with Earth shadow under tangential thrust and 
presented a solution in series expansion with respect to the eccentricity for the equations of motion, which is valid up to eccentricities of 0.2. Similarly, Kechichian 1998c, treated the problem of optimal inclination change for quasi-circular orbits. Kechichian 1997 reformulated Edelbaum's problem with optimal control and applied it to the problem of transfers between non-coplanar, circular orbits. Kechichian 1997b, 2007 investigated the problem of low-thrust trajectory optimisation under the effect of $J_{2}$ perturbation and derived the set of dynamical and adjoint equations for the solution of optimal control problems. Note that, the differential equation are not solved in closed form but integrated numerically.

Similarly, authors from the former USSR (Evtushenko 1966, cited in Beletsky 1999) studied the integrability of the motion under continuous tangential acceleration and derived some interesting closed form solutions, for example for escape spirals.

More recently, Colombo et al. (2009) proposed a semi-analytical solution for the case of tangential thrust that was applied to the low-thrust deflection of Near Earth Objects.

Lantoine and Russell (2009, 2011), proposed an analytical solution to the classical Stark problem. The authors derived an exact, closed form solution in terms of elliptic integrals, for the orbit motion perturbed by a constant acceleration in an inertial reference frame.

Bombardelli et al. (2011) proposed a first order analytical solution based on perturbation theory for the case of constant tangential acceleration.

Zuiani et al. (2010, 2012a) presented an analytical solution in non-singular orbital elements and implemented it into a direct transcription method for optimal control called Direct Finite Perturbative Elements in Time (DFPET). The same analytical formulation was applied to the propagation of the motion of an asteroid under the effect of a low-thrust deflection action (Zuiani et al. 2012b) and to the multi-objective optimisation of low-thrust active debris removal strategies (Zuiani and Vasile 2012a).

Notable examples of long low-thrust spiralling trajectories are the ones of SMART-1 (Schoenmaekers 2001), of NASA's Dawn. The latter's objective is that of visiting two dwarf planets in the asteroid belt, Vesta and Ceres (Russell et al., 2007; Smith et al., 2013). Among planned missions, JAXA's DESTINY (Zuiani, Kawakatsu and Vasile, 2013) is noteworthy, in which the thrust-to-mass ratio is in the order of $10^{-4} \mathrm{~m} / \mathrm{s}^{2}$.

This paper extends the previous work from the authors on the derivation of a general set of first order analytical solutions in non-singular elements for the Keplerian motion under a constant control acceleration. The new set of analytical formulae, presented in this paper, now includes also the contribution of a constant inertial acceleration, constant tangential acceleration and the $J_{2}$ effect. Adding to this, the approach to the solution of the time 
equation has been revised and improved compared to the previous work. The results in this paper generalise and extend the existing solutions in the literature, offering the possibility to treat different acceleration profiles and a general set of orbital elements. It will be shown that the resulting analytical approximate solution is suitable for the fast and accurate propagation of long spiralling trajectories in which different perturbative actions are present. Moreover, an application will be presented in which the analytical solutions are used to compute the average variation of the orbit over one revolution. A simplified parameterisation of the thrusting pattern is then introduced to quickly estimate the cost of long controlled spiralling transfers, like, for example, a Geostationary Transfer Orbit (GTO) to Geostationary Earth Orbit (GEO) circularisation. This approach can be seen as a form of direct transcription that does not require the derivation the first order optimality conditions and the solution of the adjoint equations.

The paper is structured as follows. Section 2 will present the derivation of the analytical formulae. Further details can be found in the Appendix in the supplementary material available online. Section 3 will start by studying the accuracy and computational cost of the analytical formulas for integrations over one revolution period. This first analysis clarifies the relationship between accuracy and acceleration magnitude. Section 3 will then show the accuracy of the proposed formulas for integrations over several hundreds of revolutions. Section 4 shows how eclipses and discontinuous control profiles are introduced in the analytical formulation, while Section 5 will present some applications. In particular, the analytical formulas are used to implement a fast averaging technique. An example of optimised transfer from a GTO to GEO concludes Section 5.

\section{Analytical Formulae}

Let the state of a spacecraft, moving around a gravitational body with gravity constant $\mu$, be expressed in terms of non-singular equinoctial elements (Battin 1987):

$$
\begin{gathered}
a \\
P_{1}=e \sin (\Omega+\omega) \\
P_{2}=e \cos (\Omega+\omega) \\
Q_{1}=\tan \frac{i}{2} \sin \Omega \\
Q_{2}=\tan \frac{i}{2} \cos \Omega \\
L=\Omega+\omega+\theta
\end{gathered}
$$


where $a$ is the semi-major axis, $e$ is the eccentricity, $i$ is the inclination, $\Omega$ is the right ascension of the ascending node, $\omega$ is the argument of perigee and $\theta$ is the true anomaly. Then, the perturbed Keplerian motion is governed by Gauss' planetary equations:

$$
\begin{gathered}
\frac{d a}{d t}=\frac{2}{B} \sqrt{\frac{a^{3}}{\mu}}\left[\left(P_{2} \sin L-P_{1} \cos L\right) a_{r}+\Phi(L) a_{\theta}\right] \\
\frac{d P_{1}}{d t}=B \sqrt{\frac{a}{\mu}}\left[-a_{r} \cos L+\left(\frac{P_{1}+\sin L}{\Phi(L)}+\sin L\right) a_{\theta}-P_{2} \frac{Q_{1} \cos L-Q_{2} \sin L}{\Phi(L)} a_{h}\right] \\
\frac{d P_{2}}{d t}=B \sqrt{\frac{a}{\mu}}\left[a_{r} \sin L+\left(\frac{P_{2}+\cos L}{\Phi(L)}+\cos L\right) a_{\theta}+P_{1} \frac{Q_{1} \cos L-Q_{2} \sin L}{\Phi(L)} a_{h}\right] \\
\frac{d Q_{1}}{d t}=\frac{B}{2} \sqrt{\frac{a}{\mu}}\left(1+Q_{1}^{2}+Q_{2}^{2}\right) \frac{\sin L}{\Phi(L)} a_{h} \\
\frac{d Q_{2}}{d t}=\frac{B}{2} \sqrt{\frac{a}{\mu}}\left(1+Q_{1}^{2}+Q_{2}^{2}\right) \frac{\cos L}{\Phi(L)} a_{h}
\end{gathered}
$$

with:

$$
\begin{aligned}
& B=\sqrt{1-P_{1}^{2}-P_{2}^{2}}=\sqrt{1-e^{2}} \\
& \Phi(L)=1+P_{1} \sin L+P_{2} \cos L
\end{aligned}
$$

The quantities $a_{r}, a_{\theta}, a_{h}$, are the components of a perturbing acceleration in the radialtransverse-normal $(r-\theta-h)$ reference frame centred in the spacecraft. The components of the perturbing acceleration can also be expressed in terms of modulus $\varepsilon$, azimuth $\alpha$ and elevation $\beta$ as:

$$
\begin{gathered}
a_{r}=\varepsilon \cos \beta \cos \alpha \\
a_{\theta}=\varepsilon \cos \beta \sin \alpha \\
a_{h}=\varepsilon \sin \beta
\end{gathered}
$$

As shown in Zuiani et al. (2012), if one assumes that the modulus of the perturbative acceleration is small compared to the local gravitational acceleration, one can write:

$$
\frac{d t}{d L} \approx \frac{r^{2}}{h}=\sqrt{\frac{a^{3}}{\mu}} \frac{B^{3}}{\Phi^{2}(L)}
$$

where $r$ is the distance from the centre of the gravitational body and $h$ is the norm of the angular momentum. By substituting [5] into Eqs. [2] one obtains a system of equations in the longitude $L$ : 


$$
\begin{gathered}
\frac{d a}{d L}=\varepsilon \frac{2 a^{3} B^{2}}{\mu}\left[\frac{\left(P_{2} \sin L-P_{1} \cos L\right)}{\Phi^{2}(L)} \cos \alpha+\frac{1}{\Phi(L)} \sin \alpha\right] \cos \beta \\
\frac{d P_{1}}{d L}=\varepsilon \frac{B^{4} a^{2}}{\mu}\left[\left(-\frac{\cos L}{\Phi^{2}(L)} \cos \alpha+\left(\frac{P_{1}+\sin L}{\Phi^{3}(L)}+\frac{\sin L}{\Phi^{2}(L)}\right) \sin \alpha\right) \cos \beta-P_{2} \frac{Q_{1} \cos L-Q_{2} \sin L}{\Phi^{3}(L)} \sin \beta\right] \\
\frac{d P_{2}}{d L}=\varepsilon \frac{B^{4} a^{2}}{\mu}\left[\left(\frac{\sin L}{\Phi^{2}(L)} \cos \alpha+\left(\frac{P_{2}+\cos L}{\Phi^{3}(L)}+\frac{\cos L}{\Phi^{2}(L)}\right) \sin \alpha\right) \cos \beta+P_{1} \frac{Q_{1} \cos L-Q_{2} \sin L}{\Phi^{3}(L)} \sin \beta\right] \\
\frac{d Q_{1}}{d L}=\varepsilon \frac{B^{4} a^{2}}{2 \mu}\left(1+Q_{1}^{2}+Q_{2}^{2}\right) \frac{\sin L}{\Phi^{3}(L)} \sin \beta \\
\frac{d Q_{2}}{d L}=\varepsilon \frac{B^{4} a^{2}}{2 \mu}\left(1+Q_{1}^{2}+Q_{2}^{2}\right) \frac{\cos L}{\Phi^{3}(L)} \sin \beta
\end{gathered}
$$

or, in vector form, taking $\mathbf{E}=\left[a, P_{1}, P_{2}, Q_{1}, Q_{2}\right]^{T}$ :

$$
\frac{d \mathbf{E}}{d L}=\varepsilon F(\mathbf{E}, L, \alpha, \beta)
$$

As in classical Regular Perturbation theory (see, for example, Holmes (2009) or Sanders, Verhulst and Murdock (2007), or also Beletsky (1999) ) one can express the solution of [7] as an expansion in the small parameter $\varepsilon$, with respect to a set of initial conditions $\mathbf{E}_{0}=\left[a_{0}, P_{10}, P_{20}, Q_{10}, Q_{20}\right]^{T}:$

$$
\mathbf{E} \approx \mathbf{E}_{0}+\varepsilon \mathbf{E}_{1}+\varepsilon^{2} \mathbf{E}_{2}+O\left(\varepsilon^{3}\right)=\left\{\begin{array}{c}
a_{0}+\varepsilon a_{1}+\varepsilon^{2} a_{2} \\
P_{10}+\varepsilon P_{11}+\varepsilon^{2} P_{12} \\
P_{20}+\varepsilon P_{21}+\varepsilon^{2} P_{22} \\
Q_{10}+\varepsilon Q_{11}+\varepsilon^{2} Q_{12} \\
Q_{20}+\varepsilon Q_{21}+\varepsilon^{2} Q_{22}
\end{array}\right\}+O\left(\varepsilon^{3}\right)
$$

Substituting [8] into [7] and expanding the right hand side in Taylor series with respect to $\varepsilon$, one obtains:

$$
\begin{aligned}
& \frac{d \mathbf{E}_{0}}{d L}+\varepsilon \frac{d \mathbf{E}_{1}}{d L}+\varepsilon^{2} \frac{d \mathbf{E}_{2}}{d L}+\ldots=0+\left.\left[F+\varepsilon \frac{d F\left(\mathbf{E}_{0}+\varepsilon \mathbf{E}_{1}+\ldots, L, \alpha, \beta\right)}{d \varepsilon}\right]\right|_{\varepsilon=0} \varepsilon+ \\
& +\left.\left[2 \frac{d F}{d \varepsilon}+\varepsilon \frac{d^{2} F}{d \varepsilon^{2}}\right]\right|_{\varepsilon=0} \frac{\varepsilon^{2}}{2}+\ldots
\end{aligned}
$$

By collecting the terms which depend on the same powers of $\varepsilon$ and neglecting second and higher order terms, one obtains: 


$$
\begin{gathered}
\frac{d \mathbf{E}_{0}}{d L}=0 \Rightarrow \mathbf{E}_{0}=\text { const } \\
\varepsilon \frac{d \mathbf{E}_{1}}{d L}=\varepsilon F\left(\mathbf{E}_{0}, L, \alpha, \beta\right)
\end{gathered}
$$

Therefore, the first-order expansion term in $\varepsilon$ becomes:

$$
\mathbf{E}_{1}(L)=\int_{L_{0}}^{L} F\left(\mathbf{E}_{0}, \mathcal{L}, \alpha, \beta\right) d \mathcal{L}
$$

To obtain a first-order expansion for the time, let one apply a similar procedure to [5]. First, let one expand the time in the form:

$$
t=t_{0}+\varepsilon t_{1}+\varepsilon^{2} t_{2}+\varepsilon^{3} t_{3}+\ldots
$$

and define the right-hand side of [5] as $H\left(a, P_{1}, P_{2}, L\right)$, then by expanding $H$ in Taylor series as done previously, one obtains:

$$
\frac{d t_{0}}{d L}+\varepsilon \frac{d t_{1}}{d L}+\varepsilon^{2} \frac{d t_{2}}{d L}+\ldots=H\left(a_{0}, P_{10}, P_{20}, L\right)+\frac{d H}{d \varepsilon} \varepsilon+\frac{d^{2} H}{d \varepsilon^{2}} \varepsilon^{2}+\ldots
$$

and taking only the first order terms in $\mathcal{E}$, one can write:

$$
\frac{d t_{0}}{d L}+\varepsilon \frac{d t_{1}}{d L}=H\left(a_{0}, P_{10}, P_{20}, L\right)+\frac{d H}{d \mathbf{E}} \frac{d \mathbf{E}}{d \varepsilon} \varepsilon
$$

Again taking only the first order terms in $\varepsilon$, one obtains:

$$
\begin{gathered}
\frac{d t_{0}}{d L}=H\left(a_{0}, P_{10}, P_{20}, L\right) \\
\varepsilon \frac{d t_{1}}{d L}=\varepsilon\left(\left.\frac{d H}{d a}\right|_{a_{0}, P_{10}, P_{20}} a_{1}+\left.\frac{d H}{d P_{1}}\right|_{a_{0}, P_{10}, P_{20}} P_{11}+\left.\frac{d H}{d P_{2}}\right|_{a_{0}, P_{10}, P_{20}} P_{21}\right)
\end{gathered}
$$

These two equations can be integrated in $L$ to get the expressions

$$
\begin{aligned}
& t_{0}(L)=t_{00}+\int_{L_{0}}^{L} \sqrt{\frac{a_{0}^{3}}{\mu}} \frac{B_{0}^{3}}{\Phi_{0}{ }^{2}(\mathcal{L})} d \mathcal{L} \\
& t_{1}(L)=\int_{L_{0}}^{L} \sqrt{\frac{a_{0}}{\mu}} B_{0}\left\{\frac{3 B_{0}{ }^{2}}{2} \frac{a_{1}(\mathcal{L})}{\Phi_{0}{ }^{2}(\mathcal{L})}-a_{0}\left[\begin{array}{l}
\left.\left(\frac{3 P_{10}}{\Phi_{0}{ }^{2}(\mathcal{L})}+2 B_{0}{ }^{2} \frac{\sin \mathcal{L}}{\Phi_{0}{ }^{3}(\mathcal{L})}\right) P_{11}(\mathcal{L})+\right] \\
\left(\frac{3 P_{20}}{\Phi_{0}{ }^{2}(\mathcal{L})}+2 B_{0}{ }^{2} \frac{\cos \mathcal{L}}{\Phi_{0}{ }^{3}(\mathcal{L})}\right) P_{21}(\mathcal{L})
\end{array}\right]\right\} d \mathcal{L}
\end{aligned}
$$

Note that the zero-order term $t_{0}$ is not simply the time $t_{00}$ corresponding to $L_{0}$, but includes also the time variation given by the unperturbed Keplerian motion. In addition, the presence of the terms $a_{1}, P_{11}, P_{21}$ essentially implies a double integration between $L_{0}$ and $L$. In the following expressions [11] and [16] will be developed for different types of perturbative acceleration. 


\subsection{Constant Acceleration in the $r-\theta-h$ Frame}

If one assumes a constant acceleration modulus and direction in the $r-\theta-h$ frame, then the expansion [8], taking only the first order terms in $\varepsilon$, takes the following form:

$$
\begin{gathered}
a^{r \vartheta h}=a_{0}+\varepsilon^{r \vartheta h} \frac{2 B_{0}{ }^{2} a_{0}{ }^{3}}{\mu} \cos \beta\left[\left(\frac{1}{\Phi_{0}(L)}-\frac{1}{\Phi_{0}\left(L_{0}\right)}\right) \cos \alpha+I_{11} \sin \alpha\right] \\
P_{1}^{r \vartheta h}=P_{10}+\varepsilon^{r \vartheta h} \frac{B_{0}{ }^{4} a_{0}{ }^{2}}{\mu}\left[\cos \beta\left(-I_{c 2} \cos \alpha+\left(I_{13} P_{10}+I_{s 3}+I_{s 2}\right) \sin \alpha\right)-P_{20}\left(Q_{10} I_{c 3}-Q_{20} I_{s 3}\right) \sin \beta\right] \\
P_{2}^{r \vartheta h}=P_{20}+\varepsilon^{r \vartheta h} \frac{B_{0}{ }^{4} a_{0}{ }^{2}}{\mu}\left[\cos \beta\left(I_{s 2} \cos \alpha+\left(I_{13} P_{20}+I_{c 3}+I_{c 2}\right) \sin \alpha\right)+P_{10}\left(Q_{10} I_{c 3}-Q_{20} I_{s 3}\right) \sin \beta\right] \\
Q_{1}^{r \vartheta h}=Q_{10}+\varepsilon^{r \vartheta h} \frac{B_{0}{ }^{4} a_{0}{ }^{2}}{2 \mu}\left(1+Q_{1}^{2}+Q_{2}^{2}\right) I_{s 3} \sin \beta \\
Q_{2}^{r \vartheta h}=Q_{20}+\varepsilon^{r \vartheta h} \frac{B_{0}{ }^{4} a_{0}{ }^{2}}{2 \mu}\left(1+Q_{1}{ }^{2}+Q_{2}{ }^{2}\right) I_{c 3} \sin \beta
\end{gathered}
$$

where the terms $I_{c n}, I_{s n}$ and $I_{1 n}$, with $n=1,2,3$, are integrals in $L$ in the form:

$$
I_{c n}=\int_{L_{0}}^{L} \frac{\cos \mathcal{L}}{\Phi_{0}^{n}(\mathcal{L})} d \mathcal{L} ; \quad I_{s n}=\int_{L_{0}}^{L} \frac{\sin \mathcal{L}}{\Phi_{0}^{n}(\mathcal{L})} d \mathcal{L} ; \quad I_{1 n}=\int_{L_{0}}^{L} \frac{1}{\Phi_{0}^{n}(\mathcal{L})} d \mathcal{L}
$$

The function $\Phi_{0}$ is the term in [3] evaluated with $P_{1=} P_{10}$ and $P_{2=} P_{20}$. The full analytical form for these integrals is reported in the online Appendix. As for the expression of $t_{1}$ in the time equation [16], one can see, from numerical analysis, that neglecting the terms in square brackets multiplying $P_{11}$ and $P_{21}$ does not introduce a relevant error in all the cases analysed in this paper. Hence, only the term depending on $a_{l}$ is retained to give the analytical expression:

$$
t_{1}^{r \vartheta h}=3 \sqrt{\frac{a_{0}^{7}}{\mu^{3}}} B_{0}{ }^{5} \cos \beta\left(\cos \alpha I_{t 1}+\sin \alpha I_{t 2}\right)
$$

where:

$$
I_{t 1}=\int_{L_{0}}^{L} \frac{1}{\Phi_{0}{ }^{2}(\mathcal{L})}\left(\frac{1}{\Phi_{0}(\mathcal{L})}-\frac{1}{\Phi_{0}\left(L_{0}\right)}\right) d \mathcal{L} \quad I_{t 2}=\int_{L_{0}}^{L} \frac{I_{11}(\mathcal{L})}{\Phi_{0}{ }^{2}(\mathcal{L})} d \mathcal{L}
$$

The full analytical expansions for these integrals are found in the online Appendix.

\subsection{Constant Inertial Acceleration}

A constant acceleration in the inertial reference frame can be expressed, in the $r-\theta-h$ frame, as a function of the longitude $L$ :

$$
a_{r}^{I n}=\varepsilon^{I n} \cos \beta_{0} \cos \left(\gamma_{0}-L\right) \quad a_{\theta}{ }^{I n}=\varepsilon^{I n} \cos \beta_{0} \sin \left(\gamma_{0}-L\right) \quad a_{h}{ }^{I n}=\varepsilon^{I n} \sin \beta_{0}
$$


where $\gamma_{0}$ derives from the initial acceleration azimuth $\alpha_{0}$ at $L_{0}$, as:

$$
\gamma_{0}=\alpha_{0}+L_{0}
$$

Note that the initial azimuth $\alpha_{0}$ and elevation $\beta_{0}$ are defined with respect to the $r-\theta-h$ frame at $L_{0 .}$ By substituting [21] into [6], and after some manipulation, one can obtain an expression analogous to [17]:

$$
\begin{gathered}
a^{I n}=a_{0}+\varepsilon^{I n} \frac{2 B_{0}{ }^{2} a_{0}{ }^{3}}{\mu} \cos \beta_{0}\left[-\left(P_{10} I_{12}+I_{s 2}\right) \cos \gamma_{0}+\left(P_{20} I_{12}+I_{c 2}\right) \sin \gamma_{0}\right] \\
P_{1}^{I n}=P_{10}+\varepsilon^{I n} \frac{B_{0}{ }^{4} a_{0}{ }^{2}}{\mu}\left\{\operatorname { c o s } \beta _ { 0 } \left[-\left(P_{10} I_{s 3}+I_{12}+I_{2 s 3}\right) \cos \gamma_{0}+\right.\right. \\
\left.\left.+\left(P_{10} I_{c 3}+I_{1 c 1 s 3}\right) \sin \gamma_{0}\right]-\sin \beta_{0} P_{20}\left(Q_{10} I_{c 3}-Q_{20} I_{s 3}\right)\right\} \\
P_{2}^{I n}=P_{20}+\varepsilon^{I n} \frac{B_{0}{ }^{4} a_{0}{ }^{2}}{\mu}\left\{\operatorname { c o s } \beta _ { 0 } \left[-\left(P_{20} I_{s 3}+I_{1 c 1 s 3}\right) \cos \gamma_{0}+\right.\right. \\
\left.\left.+\left(P_{20} I_{c 3}+I_{12}+I_{2 c 3}\right) \sin \gamma_{0}\right]+\sin \beta_{0} P_{10}\left(Q_{10} I_{c 3}-Q_{20} I_{s 3}\right)\right\} \\
Q_{1}^{I n}=Q_{10}+\varepsilon^{I n} \frac{B_{0}{ }^{4} a_{0}{ }^{2}}{2 \mu}\left(1+Q_{1}^{2}+Q_{2}{ }^{2}\right) \sin \beta_{0} I_{s 3} \\
Q_{2}{ }^{I n}=Q_{20}+\varepsilon^{I n} \frac{B_{0}{ }^{4} a_{0}{ }^{2}}{2 \mu}\left(1+Q_{1}^{2}+Q_{2}{ }^{2}\right) \sin \beta_{0} I_{c 3}
\end{gathered}
$$

where the integral terms $I_{1 c 1 s 3}, I_{2 c 3} I_{2 s 3}$ are given in the online Appendix. Similarly, the first-order perturbative term in the time equation translates into:

$$
t_{1}^{I n}=3 \sqrt{\frac{a_{0}^{7}}{\mu^{3}}} B_{0}^{5} \cos \beta_{0}\left\{-\frac{\cos \gamma_{0}}{P_{20}}\left[\left(I_{13}+P_{10} I_{s 3}\right)-\frac{\left(1+P_{10} \sin L_{0}\right)}{\Phi_{0}\left(L_{0}\right)} I_{12}\right]+\sin \gamma_{0}\left(I_{s 3}-\frac{\sin L_{0}}{\Phi_{0}\left(L_{0}\right)} I_{12}\right)\right\}
$$

\subsection{Constant Tangential Acceleration}

A constant acceleration along the tangential direction can be expressed, in the $r-\theta-h$ frame, as a function of the longitude $L$ :

$$
a_{r}^{t}=\varepsilon^{t} \frac{P_{2} \sin L-P_{1} \cos L}{D} \quad a_{\theta}{ }^{t}=\varepsilon^{t} \frac{1+P_{1} \sin L+P_{2} \cos L}{D} \quad a_{h}^{t}=0
$$

where $D$ is expressed as:

$$
D=\sqrt{1+P_{1}^{2}+P_{2}^{2}+2\left(P_{1} \sin L+P_{2} \cos L\right)}
$$

Again, by substituting [25] into [6] one obtains: 


$$
\begin{gathered}
a^{t}=a_{0}+\varepsilon^{t} \frac{2 B_{0}{ }^{2} a_{0}{ }^{3}}{\mu} \int_{L_{0}}^{L} \frac{1}{D_{0}(\mathcal{L})}\left(\frac{2}{\Phi_{0}(\mathcal{L})}-\frac{B_{0}{ }^{2}}{\Phi_{0}(\mathcal{L})^{2}}\right) d \mathcal{L} \\
P_{1}^{t}=P_{10}+\varepsilon^{t} \frac{B_{0}{ }^{4} a_{0}{ }^{2}}{\mu} \int_{L_{0}}^{L} \frac{2\left(P_{10}+\sin \mathcal{L}\right)}{D_{0}(\mathcal{L}) \Phi_{0}(\mathcal{L})^{2}} d \mathcal{L} \\
P_{2}^{t}=P_{20}+\varepsilon^{t} \frac{B_{0}{ }^{4} a_{0}{ }^{2}}{\mu} \int_{L_{0}}^{L} \frac{2\left(P_{20}+\cos \mathcal{L}\right)}{D_{0}(\mathcal{L}) \Phi_{0}(\mathcal{L})^{2}} d \mathcal{L} \\
Q_{1}{ }^{t}=Q_{10} \\
Q_{2}{ }^{t}=Q_{20}
\end{gathered}
$$

$D_{0}$ is simply $D$ evaluated with $P_{1}=P_{10}$ and $P_{2}=P_{20}$. The three integral terms are more conveniently expressed with respect to the true anomaly $\theta$ and eccentricity $e$, assuming that $\theta \approx L-\left(\Omega_{0}+\omega_{0}\right):$

$$
\begin{gathered}
\int_{L_{0}}^{L} \frac{1}{D_{0}(\mathcal{L})}\left(\frac{2}{\Phi_{0}(\mathcal{L})}-\frac{B_{0}{ }^{2}}{\Phi_{0}(\mathcal{L})^{2}}\right) d \mathcal{L}=\int_{\theta_{0}}^{\theta} \frac{\sqrt{1+e_{0}{ }^{2}+2 e_{0} \cos \vartheta}}{\left(1+e_{0} \cos \vartheta\right)^{2}} d \vartheta=I_{a} \\
\int_{L_{0}}^{L} \frac{P_{10}+\sin \mathcal{L}}{D_{0}(\mathcal{L}) \Phi_{0}(\mathcal{L})^{2}} d \mathcal{L}=\sin \left(\Omega_{0}+\omega_{0}\right) I_{P 1}+\cos \left(\Omega_{0}+\omega_{0}\right) I_{P 2} \\
\int_{L_{0}}^{L} \frac{P_{20}+\cos \mathcal{L}}{D_{0}(\mathcal{L}) \Phi_{0}(\mathcal{L})^{2}} d \mathcal{L}=\cos \left(\Omega_{0}+\omega_{0}\right) I_{P 1}-\sin \left(\Omega_{0}+\omega_{0}\right) I_{P 2}
\end{gathered}
$$

where

$$
\begin{aligned}
& I_{P 1}=\int_{\theta_{0}}^{\theta} \frac{e_{0}+\cos \vartheta}{\sqrt{1+e_{0}^{2}+2 e_{0} \cos \vartheta}\left(1+e_{0} \cos \vartheta\right)^{2}} d \vartheta \\
& I_{P 2}=\int_{\theta_{0}}^{\theta} \frac{\sin \vartheta}{\sqrt{1+e_{0}^{2}+2 e_{0} \cos \vartheta}\left(1+e_{0} \cos \vartheta\right)^{2}} d \vartheta
\end{aligned}
$$

The primitives of $I_{a}, I_{P 1}$ and $I_{P 2}$ are available in closed form and are reported in the online Appendix. Note that in [16] and [17] of the online Appendix there are terms that represent incomplete elliptic integrals of the first and second kind, represented as $F$ and $E$ respectively. In this work, $E$ and $F$ are conveniently evaluated numerically using Carlson's Duplication Algorithms (Carlson 1979). Rearranging the terms in [27], one obtains (omitting the expressions for $Q_{1}$ and $Q_{2}$ ):

$$
\begin{gathered}
a^{t}=a_{0}+\varepsilon^{t} \frac{2 B_{0}{ }^{2} a_{0}{ }^{3}}{\mu} I_{a} \\
P_{1}^{t}=P_{10}+\varepsilon^{t} 2 \frac{B_{0}{ }^{4} a_{0}{ }^{2}}{\mu}\left(\sin \left(\Omega_{0}+\omega_{0}\right) I_{P 1}+\cos \left(\Omega_{0}+\omega_{0}\right) I_{P 2}\right) \\
P_{2}^{t}=P_{20}+\varepsilon^{t} 2 \frac{B_{0}{ }^{4} a_{0}{ }^{2}}{\mu}\left(\cos \left(\Omega_{0}+\omega_{0}\right) I_{P 1}+\sin \left(\Omega_{0}+\omega_{0}\right) I_{P 2}\right)
\end{gathered}
$$


In the case of tangential thrust, in the first order term of the time equation [16], one has terms that depend on the above-mentioned elliptic integrals, which appear in [28]. After some manipulation, one would obtain:

$$
\begin{aligned}
& t_{1}^{t}(\theta)=\frac{B_{0}^{5} a_{0}^{3.5}}{\mu^{1.5}} \int_{\theta_{0}}^{\theta} \frac{1}{\Phi_{0}{ }^{2}(\vartheta)}\left\{3 I_{a}(\vartheta)-\right. \\
& \left.-\frac{1}{\Phi_{0}(\vartheta)_{0}}\left[\begin{array}{l}
\left(6 e_{0}+2 e_{0}^{2} \cos \vartheta+4 \cos \vartheta\right) I_{P 1}(\vartheta)+ \\
4 \sin \vartheta\left(1-e_{0}^{2}\right) I_{P 2}(\vartheta)
\end{array}\right]\right\} d \vartheta
\end{aligned}
$$

Expanding the terms in the integral, for example, considering only $I_{a}$ (as in Eq. [16] of the online Appendix), the following integral emerges:

$$
\int_{\theta_{0}}^{\theta} \frac{1}{\left(1+e_{0} \cos \vartheta\right)^{2}}\left[\frac{1}{1-e_{0}} E\left(\frac{\vartheta}{2}, \frac{4 e_{0}}{\left(1+e_{0}\right)^{2}}\right)+\frac{1}{1+e_{0}} F\left(\frac{\vartheta}{2}, \frac{4 e_{0}}{\left(1+e_{0}\right)^{2}}\right)-\frac{\sqrt{1+e_{0}^{2}+2 e_{0} \cos \vartheta} e_{0} \sin \vartheta}{\left(1-e_{0}\right)^{2}\left(1+e_{0} \cos \vartheta\right)}\right] d \vartheta
$$

Note that the terms $\int_{\theta_{0}}^{\theta} \frac{E\left(\frac{\vartheta}{2}, \frac{4 e_{0}}{\left(1+e_{0}\right)^{2}}\right)}{\left(1+e_{0} \cos \vartheta\right)^{2}} d \vartheta, \int_{\theta_{0}}^{\theta} \frac{F\left(\frac{\vartheta}{2}, \frac{4 e_{0}}{\left(1+e_{0}\right)^{2}}\right)}{\left(1+e_{0} \cos \vartheta\right)^{2}} d \vartheta$ are not available in

closed form. Similar considerations apply to the terms in [31] depending on $I_{P 1}$ and $I_{P 2}$. Therefore, in this case, it has been chosen to numerically integrate the expression for $t_{l}$ in [16] with a quadrature method. Numerical tests have shown that, by setting the number of integration nodes at 6 per revolution, adequate accuracy is achieved.

\section{4. $J_{2}$ Perturbation}

The inhomogeneous gravity field of the Earth is usually modelled as a series expansion of harmonic functions whose coefficients are experimentally derived in order to match the observed motion of satellites. The strongest contribution to the motion of an Earth-orbiting satellite is given by the first zonal harmonic term, also known as $J_{2}$ term (Battin 1987). The effect of the $J_{2}$ term can be expressed as a perturbative term in the Gauss variational equations. In particular, the components of the $J_{2}$ perturbation in the $r-\theta-h$ frame can be expressed as (Kechichian 1997): 


$$
\begin{gathered}
a_{r}^{J_{2}}=\frac{3 \mu J_{2} R^{2}}{2 B^{8} a^{4}}\left[\frac{12}{G^{2}}\left(Q_{1} \cos L-Q_{2} \sin L\right)^{2}-1\right] \Phi^{4}(L) \\
a_{\theta}^{J_{2}}=\frac{12 \mu J_{2} R^{2}}{B^{8} G^{2} a^{4}}\left(Q_{2} \cos L+Q_{1} \sin L\right)\left(Q_{1} \cos L-Q_{2} \sin L\right) \Phi^{4}(L) \\
a_{h}^{J_{2}}=\frac{6 \mu J_{2} R^{2}}{B^{8} G^{2} a^{4}}\left(Q_{1} \cos L-Q_{2} \sin L\right)\left(1-Q_{1}{ }^{2}-Q_{2}{ }^{2}\right) \Phi^{4}(L)
\end{gathered}
$$

where $R$ is the planetary radius and $G$ is:

$$
G=1+Q_{1}^{2}+Q_{2}^{2}
$$

By substituting [33] into [6], and with the procedure previously described, one can write the first-order variation of the equinoctial elements due to the $J_{2}$ perturbation. In a compact form, this can be expressed as:

$$
\begin{gathered}
a^{J_{2}}=a_{0}+\varepsilon^{J_{2}} \frac{a_{0}}{4 G_{0}{ }^{2} B_{0}{ }^{6}} I_{J a}\left(L_{0}, L, P_{10}, P_{20}, Q_{10}, Q_{20}\right) \\
P_{1}^{J_{2}}=P_{10}+\varepsilon^{J_{2}} \frac{1}{8 G_{0}{ }^{2} B_{0}{ }^{4}} I_{J P_{1}}\left(L_{0}, L, P_{10}, P_{20}, Q_{10}, Q_{20}\right) \\
P_{2}^{J_{2}}=P_{20}+\varepsilon^{J_{2}} \frac{1}{8 G_{0}{ }^{2} B_{0}{ }^{4}} I_{J P_{2}}\left(L_{0}, L, P_{10}, P_{20}, Q_{10}, Q_{20}\right) \\
Q_{1}^{J_{2}}=Q_{10}+\varepsilon^{J_{2}} \frac{1-Q_{10}{ }^{2}-Q_{20}{ }^{2}}{4 G_{0} B_{0}{ }^{2}} I_{J Q_{1}}\left(L_{0}, L, P_{10}, P_{20}, Q_{10}, Q_{20}\right) \\
Q_{2}^{J_{2}}=Q_{20}+\varepsilon^{J_{2}} \frac{1-Q_{10}{ }^{2}-Q_{20}{ }^{2}}{4 G_{0} B_{0}{ }^{4}} I_{J Q_{2}}\left(L_{0}, L, P_{10}, P_{20}, Q_{10}, Q_{20}\right)
\end{gathered}
$$

where $\varepsilon^{J_{2}}$ is defined as $\varepsilon^{J_{2}}=J_{2} R^{2} a_{0}{ }^{-2} . G_{0}$ is $G$, as in [34], evaluated with $Q_{1=} Q_{10}$ and $Q_{2}=Q_{20}$. The integral terms are represented as: 


$$
\begin{aligned}
& I_{J a}=\int_{L_{0}}^{L} \Phi_{0}^{2}(\mathcal{L})\left(\begin{array}{l}
12\left(P_{20} \sin \mathcal{L}-P_{10} \cos \mathcal{L}\right)\left(12\left(Q_{20} \sin \mathcal{L}-Q_{10} \cos \mathcal{L}\right)^{2}-G_{0}^{2}\right) \\
+96 \Phi_{0}(\mathcal{L})\left(Q_{10} \cos \mathcal{L}-Q_{20} \sin \mathcal{L}\right)\left(Q_{20} \cos \mathcal{L}+Q_{10} \sin \mathcal{L}\right)
\end{array}\right) d \mathcal{L} \\
& I_{J P_{1}}=\int_{L_{0}}^{L} \Phi_{0}(\mathcal{L})\left(\begin{array}{l}
12 \cos \mathcal{L} \Phi_{0}(\mathcal{L})\left(G_{0}^{2}-12\left(Q_{20} \sin \mathcal{L}-Q_{10} \cos \mathcal{L}\right)^{2}\right)+ \\
96\left(P_{10}+\left(1+\Phi_{0}(\mathcal{L})\right) \sin \mathcal{L}\right)\left(Q_{10} \cos \mathcal{L}-Q_{20} \sin \mathcal{L}\right)\left(Q_{20} \cos \mathcal{L}+Q_{10} \sin \mathcal{L}\right)+ \\
-48 P_{20}\left(Q_{10} \cos \mathcal{L}-Q_{20} \sin \mathcal{L}\right)^{2}\left(2-G_{0}\right)
\end{array}\right) d \mathcal{L} \\
& I_{J P_{2}}=\int_{L_{0}}^{L} \Phi_{0}(\mathcal{L})\left(\begin{array}{l}
12 \sin \mathcal{L} \Phi_{0}(\mathcal{L})\left(12\left(Q_{20} \sin \mathcal{L}-Q_{10} \cos \mathcal{L}\right)^{2}-G_{0}{ }^{2}\right)+ \\
96\left(P_{20}+\left(1+\Phi_{0}(\mathcal{L})\right) \cos \mathcal{L}\right)\left(Q_{10} \cos \mathcal{L}-Q_{20} \sin \mathcal{L}\right)\left(Q_{20} \cos \mathcal{L}+Q_{10} \sin \mathcal{L}\right)+ \\
48 P_{10}\left(Q_{10} \cos \mathcal{L}-Q_{20} \sin \mathcal{L}\right)^{2}\left(2-G_{0}\right)
\end{array}\right) d \mathcal{L} \\
& I_{J Q_{1}}=\int_{L_{0}}^{L} 12 \Phi_{0}(\mathcal{L})\left(Q_{10} \cos \mathcal{L}-Q_{20} \sin \mathcal{L}\right) \sin \mathcal{L} d \mathcal{L} \\
& I_{J Q_{2}}=\int_{L_{0}}^{L} 12 \Phi_{0}(\mathcal{L})\left(Q_{10} \cos \mathcal{L}-Q_{20} \sin \mathcal{L}\right) \cos \mathcal{L} d \mathcal{L}
\end{aligned}
$$

Their analytical expressions are reported in the online Appendix. Note that there is no linear component in $L$ in the expression of $I_{J a}$ (given in Eq. [19] in the online Appendix), confirming the known result that $J_{2}$ is not inducing any secular variation of the semi-major axis and thus the energy. There is, on the other hand, a short-term periodic variation of $a$ over one orbital revolution. The remaining equinoctial elements (see Equations [20], [21], [22] and [23] of the online Appendix), present both a short-term periodic variation and a secular one, which is linear with respect to $L$.

\subsection{Superimposition of Perturbations}

It has been assumed that the perturbing acceleration is small, and consequently that the variation of the orbital elements induced by this perturbation will similarly be small. For example, one can assume that the variation of the orbital plane due to $J_{2}$ and out-of-plane thrust will be small and therefore the Equation [21] for the inertial acceleration is still applicable. In this sense, it is also possible to linearly superimpose the four analytical solutions shown in the previous sections to obtain an analytical expansion for the case in which these perturbations are acting together: 


$$
\begin{aligned}
& a^{\text {tot }}=a_{0}+\varepsilon^{r \vartheta h} a_{1}^{r \vartheta h}+\varepsilon^{I n} a_{1}^{I n}+\varepsilon^{t} a_{1}^{t}+\varepsilon^{J_{2}} a_{1}^{J_{2}} \\
& P_{1}^{\text {tot }}=P_{10}+\varepsilon^{r \vartheta h} P_{11}^{r \% h}+\varepsilon^{\text {In }} P_{11}{ }^{\text {In }}+\varepsilon^{t} P_{11}{ }^{t}+\varepsilon^{J_{2}} P_{11}^{J_{2}} \\
& P_{2}^{\text {tot }}=P_{20}+\mathcal{E}^{r \vartheta h} P_{21}{ }^{r \vartheta h}+\varepsilon^{I n} P_{21}{ }^{I n}+\varepsilon^{t} P_{21}{ }^{t}+\varepsilon^{J_{2}} P_{21}{ }^{J_{2}} \\
& Q_{1}{ }^{\text {tot }}=Q_{10}+\varepsilon^{r \vartheta h} Q_{11}{ }^{r \vartheta h}+\varepsilon^{I n} Q_{11}{ }^{I n}+\varepsilon^{t} Q_{11}{ }^{t}+\varepsilon^{J_{2}} Q_{11}{ }^{J_{2}} \\
& Q_{2}{ }^{\text {tot }}=Q_{20}+\varepsilon^{r \vartheta h} Q_{21}{ }^{r \vartheta h}+\varepsilon^{I n} Q_{21}{ }^{I n}+\varepsilon^{t} Q_{21}{ }^{t}+\varepsilon^{J_{2}} Q_{21}{ }^{J_{2}} \\
& t^{t o t}=t_{00}+\varepsilon^{r \vartheta h} t_{1}^{r \vartheta h}+\varepsilon^{I n} t_{1}^{I n}+\varepsilon^{t} t_{1}^{t}+\varepsilon^{J_{2}} t_{1}^{J_{2}}
\end{aligned}
$$

\section{Accuracy of the Analytical Expansions}

This section contains an analysis of the accuracy and computational cost of the analytical formulae presented in the previous section. Accuracy and computational cost are evaluated against a numeric integration of [6] with different integration schemes and accuracies. An initial elliptical orbit, whose orbital parameters are given in Table 1, is propagated under the combined effect of the $J_{2}$ perturbation, an acceleration along the tangential direction of $10^{-4}$ $\mathrm{m} / \mathrm{s}^{2}$ and an inertial acceleration of $10^{-6} \mathrm{~m} / \mathrm{s}^{2}$ (equivalent to solar radiation pressure acting on a spacecraft with an area to mass ratio of $1 / 4.56 \mathrm{~m}^{2} / \mathrm{kg}$ at $\left.1 \mathrm{AU}\right)$.

\begin{tabular}{|c|c|c|c|c|c|}
\hline$a$ & $e$ & $i$ & $\Omega$ & $\omega$ & $\theta$ \\
\hline $24478 \mathrm{~km}$ & 0.73 & $6^{\circ}$ & $0^{\circ}$ & $0^{\circ}$ & $0^{\circ}$ \\
\hline
\end{tabular}

Table 1: GTO orbital parameters

At first, the motion is propagated for an arc-length up to $2 \pi$ and the performance of the analytical formulae is evaluated against three numerical integration schemes. This first analysis provides an evaluation of the suitability of the analytical formulae as fast integration method to be used in the orbit averaging that will be presented in section 5 . The numerical integration schemes are: a Gauss-Legendre quadrature formula with a number of nodes between 4 and 24, a Modified Euler method with a number of equally spaced steps between 4 and 16, and an $8^{\text {th }}$-order Runge-Kutta method with 13 steps (RK8(7)13) (Prince and Dormand 1981). Note that the Modified Euler evaluates the integrand function twice per step, therefore the number of function evaluations is double the number of steps. For each method, the integration error is computed as the difference between the analytical formulae and a numerical integration performed with MATLAB ${ }^{\circledR}$ ode113, implementing an Adams-Bashfort predictor-corrector method, with relative and absolute tolerances equal to $10^{-13}$. 


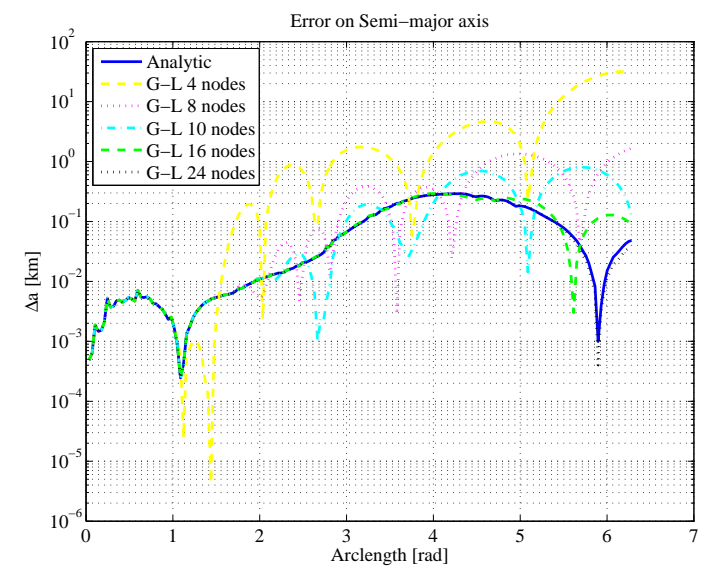

a)

Fig. 1: Comparison with numerical integration: error on semi-major axis. a) Gauss-Legendre b) Modified Euler and Runge-Kutta.

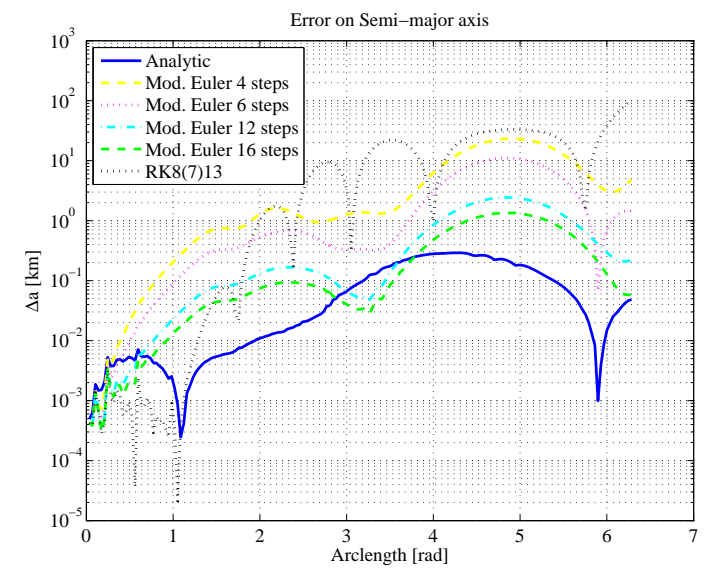

b)

Fig. 1a shows the error on the semi-major axis. One can see that the Gauss-Legendre integration has a rather noisy behaviour already for short arc-lengths; only the case with 24 nodes shows comparable accuracy to the analytical propagation. Moreover it has to be noted that Gauss-Legendre quadrature will, at best, have the same accuracy of the analytical step since it is numerically calculating the same integral forms. Fig. $1 \mathrm{~b}$ shows a similar comparison with the Modified Euler and RK8(7)13 methods and leads to analogous conclusions. The Modified Euler integrator gives good results only with a high number of steps. The RK8(7)13 scheme is extremely accurate for short arc-length, but as this increases, the numerical integral quickly diverges from the true solution. Fig. 2a, Fig. 2b and Fig. 3a plot the summary of the maximum errors on semi-major axis, $P_{1}$ and time respectively, and clearly show the better accuracy of the analytical propagation. Only Gauss Legendre with 24 approaches the same level of performance but its computational cost, as shown in Fig. 3b, is almost three times higher.
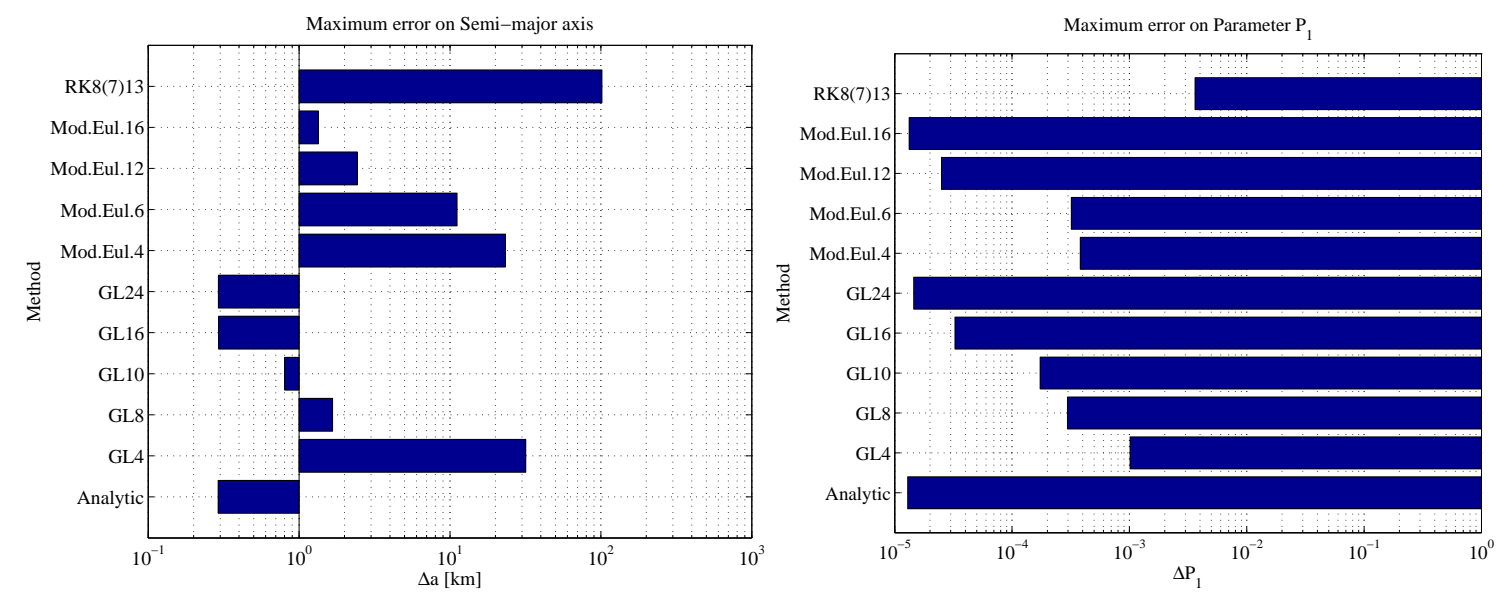

a)

b)

Fig. 2: Comparison with numerical integration: maximum error on: a) semi-major axis b) $P_{l}$. 


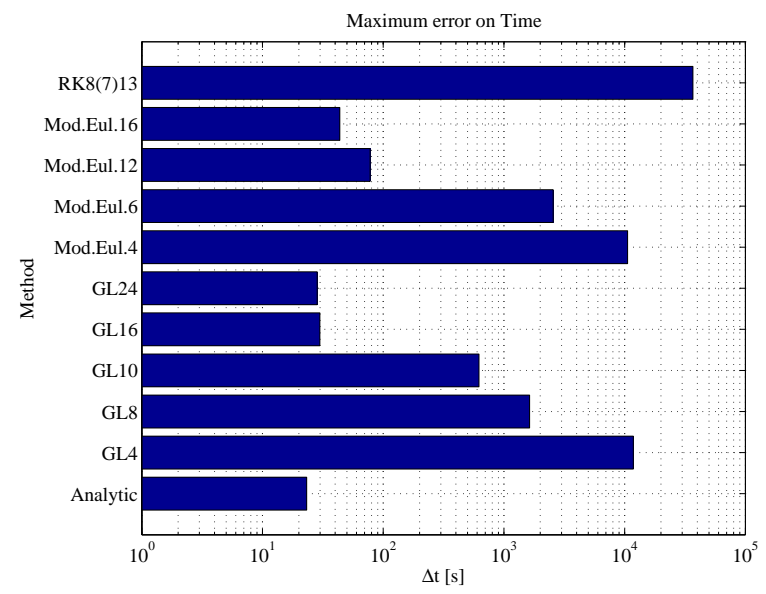

a)

Fig. 3: Comparison with numerical integration: a) maximum error on time b) computational cost.

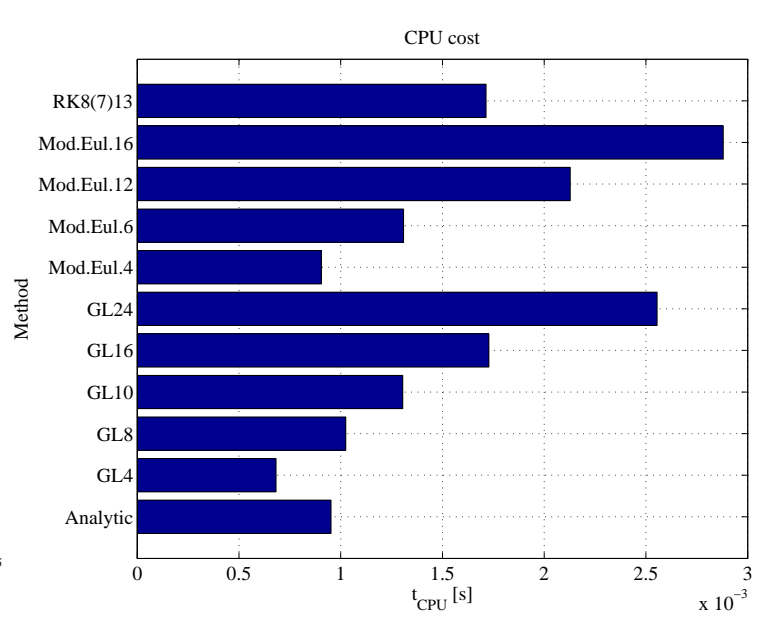

b)

Note that, in this comparison, the analytical formulae require the computation of relatively expensive elliptic integrals. If a constant acceleration in a $r-\theta-h$ frame is considered instead the computational cost is about a quarter of that of a constant tangential acceleration.

With a generic control profile as described in Section 4, at each revolution one would have to perform as many analytical propagations as the number of thrusting and coasting arcs. One can argue that in such a case, the cost of the analytical propagation might no longer be advantageous compared to other methods. In another test, the motion is propagated for one revolution under the effect of a tangential thrust for an arc of length $2 / 3 \pi$, followed by a coasting arc of length $\pi / 3$ (with $J_{2}$ only), another propelled arc of length $2 / 3 \pi$, and finally another coasting arc of length $\pi / 3$. Therefore, there are 4 separate propagations to be performed with the analytical formulae. This is an interesting case as it mimics the case of a bang-zero-bang control, as described in Section 4. The accuracy and computational cost of the analytical solution are compared to a fully numerical integration performed with GaussLegendre quadrature. Two slightly different techniques are tested: in the first one, the motion is propagated without splitting the integration interval in the three discontinuous points; in the second one, the orbit is split into four intervals (as is done for the analytical propagation) and Gauss-Legendre quadrature is applied to each of them. The number of nodes for each interval is chosen such that the number of nodes is comparable to the first case when a single integration is performed. In particular, quadrature formulas 16 and 24 nodes have been tested for the single interval case; for the split interval case, three combinations of $5+5+5+5$, $7+3+7+3$ and $6+6+6+6$ nodes were tested.

Fig. 4 and Fig. 5 show the maximum error on semi-major axis and time. Even in this case, the analytical propagation is more accurate than the numerical quadrature. Only the Gauss- 
Legendre quadrature with $6+6+6+6$ nodes displays a comparable or lower error on the semimajor axis (see Fig. 4a), although it fares worse in the other cases. In terms of computational cost (see Fig. 5b) only Gauss-Legendre with 16 nodes is cheaper than the analytical propagation, while the others are more expensive.
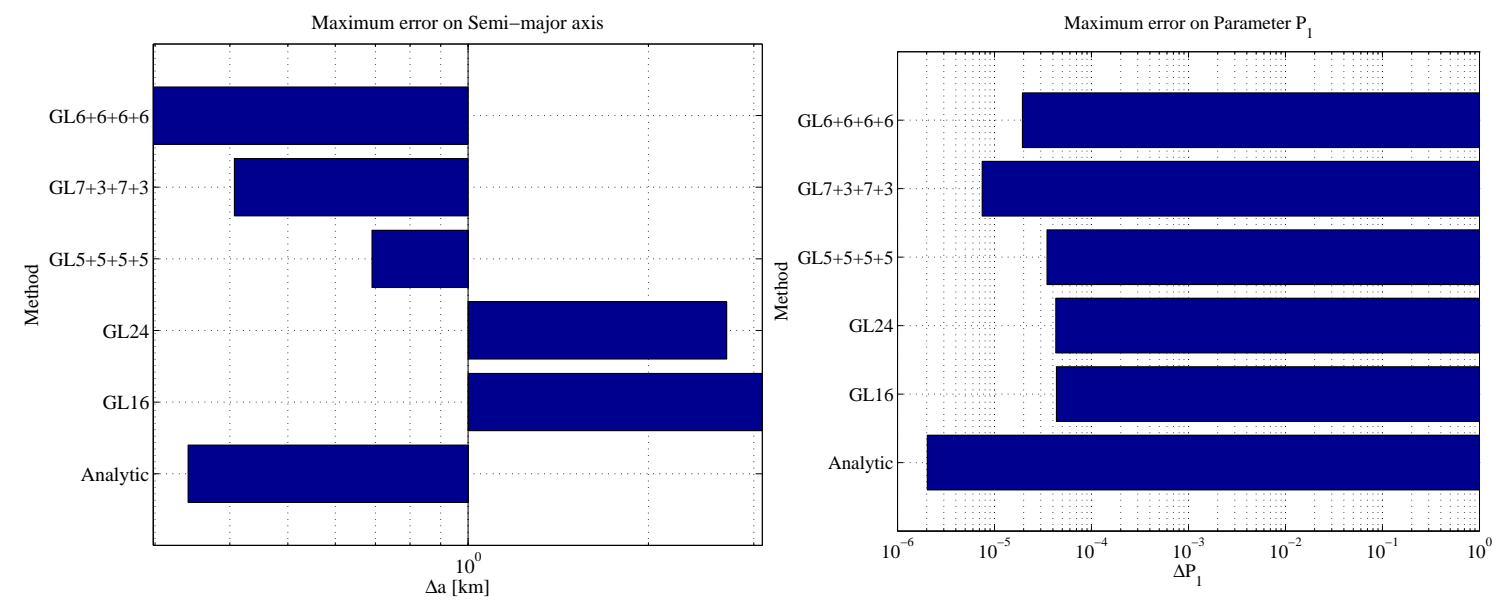

a)

b)

Fig. 4: Comparison with numerical integration on discontinuous arcs: a) maximum error on semi-major axis b) maximum error on time.

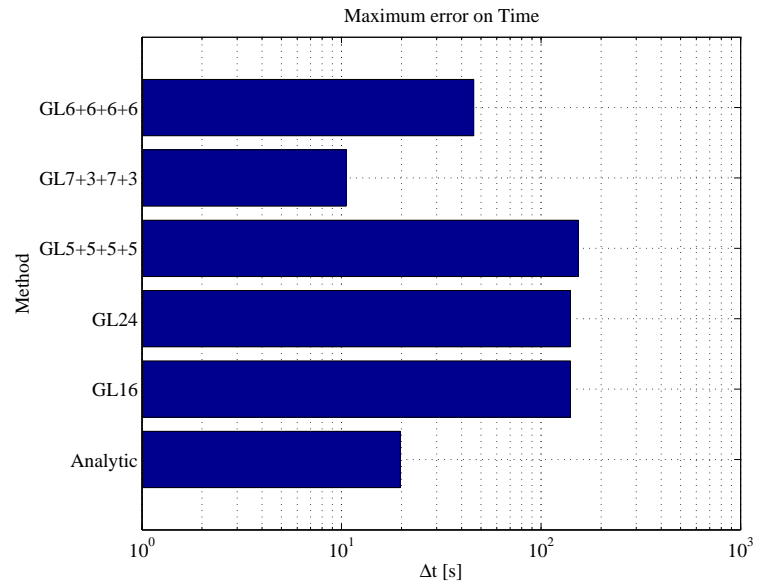

a)

Fig. 5: Comparison with numerical integration on discontinuous arcs: a) maximum error on time b) computational cost.

\subsection{Accuracy vs. Initial Semi-major Axis and $\varepsilon$}

In this section, the behaviour of the error of the analytical formulae is investigated with respect to the initial semi-major axis and the magnitude of the perturbing acceleration. This is assessed by computing the error accumulated over one orbit as a function of the magnitude of the perturbative acceleration $\varepsilon$ and of the semi-major axis $a_{0}$ of the initial orbit. A number of initial Earth-centred orbits with eccentricity 0.7 and variable $a_{0}$ were propagated with 
different $\varepsilon$, aligned along the tangential direction. Although here, for simplicity, only the tangential acceleration case is considered, the same considerations are applicable to the other perturbative accelerations. Fig. 6a shows the error on the semi-major axis relative to $a_{0}$, as a function of $a_{0}$ itself and $\varepsilon$. One can see that for a large initial semi-major axis and $\varepsilon=10^{-3}$ $\mathrm{m} / \mathrm{s}^{2}$ the error grows above $1 \%$. However one should consider that $10^{-3} \mathrm{~m} / \mathrm{s}^{2}$ is a performance level hardly attainable with current electric propulsion technology. If the acceleration is instead in the range of $10^{-4}$ to $10^{-6} \mathrm{~m} / \mathrm{s}^{2}$, the resulting propagation error remains below 0.001 for relatively large orbits with semi-major axis up to about $10^{5} \mathrm{~km}$. Note also that all orbits in the LEO to GEO class are integrated very accurately, with a relative error lower than $10^{-5}$.

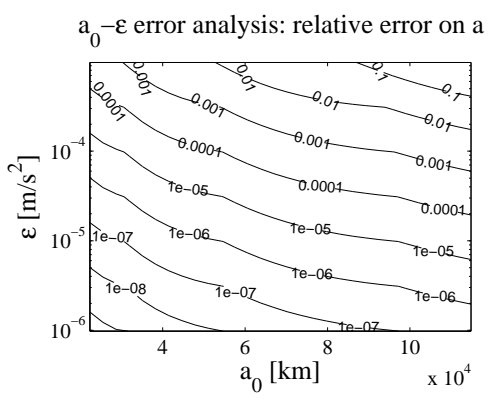

a)

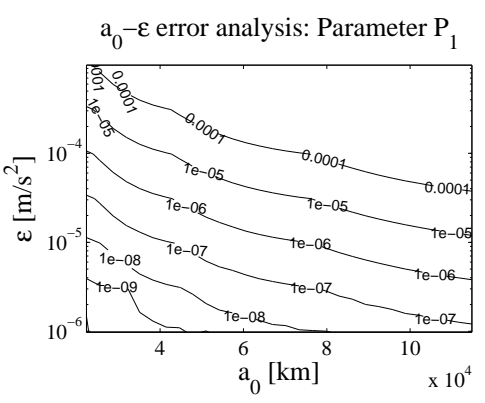

b)

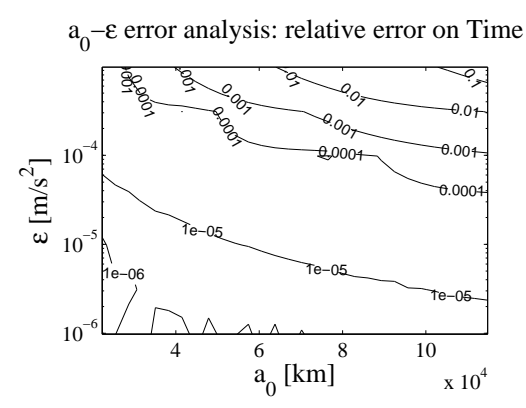

c)

Fig. 6 a): Relative error on $a$ over one revolution w.r.t. $a_{0}$ and $\varepsilon$. b): Error on $P_{1}$ over one revolution w.r.t. $a_{\theta}$ and $\varepsilon . c$ ): Relative error on time over one revolution w.r.t. $a_{0}$ and $\varepsilon$.

A similar behaviour can be observed in Fig. $6 \mathrm{~b}$ for $P_{1}$ and in Fig. $6 \mathrm{c}$ for the time $t$. The former is closely related to the orbit eccentricity and therefore it is desirable to keep the error per orbit below $10^{-5}$ to $10^{-6}$ which, as shown in the graph, can be attained in most cases except for high $a_{0}$, large $\varepsilon$ combinations. Fig. $6 \mathrm{c}$ shows the error on time divided by the period of the initial osculating orbit and one can see that the perturbed orbit duration is also computed very accurately with the error being just a fraction of the total duration.

\subsection{Propagation Over Long Arcs}

In this section, the speed and accuracy of the analytical first-order expansions is further assessed on the propagation of long arcs. For each of the four expansion formulae, an initial orbit around the Earth, with orbital parameters given in Table 2, is propagated analytically along an arc of length equal to 20 full revolutions. The difference between the value of the orbital elements along the propagated arc and the result of a full numerical integration of Gauss' variational equations is then computed to give the errors difference $\Delta a, \Delta P_{1}, \Delta P_{2}$, $\Delta Q_{1}, \Delta Q_{2}, \Delta t$. Both propagations are run in MATLAB and the numerical integration is performed with ode113 with relative and absolute tolerances set to $10^{-13}$. 


\begin{tabular}{|c|c|c|c|c|c|}
\hline$a$ & $e$ & $i$ & $\Omega$ & $\omega$ & $\theta$ \\
\hline $7500 \mathrm{~km}$ & 0.1 & $6^{\circ}$ & $0^{\circ}$ & $10^{\circ}$ & $0^{\circ}$ \\
\hline
\end{tabular}

Table 2: Initial orbit parameters

The following test assesses the accuracy and speed of the formulae in the worst case of combined pertubations, as described in Section 2.5. The modulus of the acceleration in the $r$ $\theta$ - $h$ frame is $\varepsilon=10^{-4} \mathrm{~m} / \mathrm{s}^{2}$, with $\alpha=\pi / 2$ and $\beta=\pi / 6$. The inertial and tangential components have the same modulus.
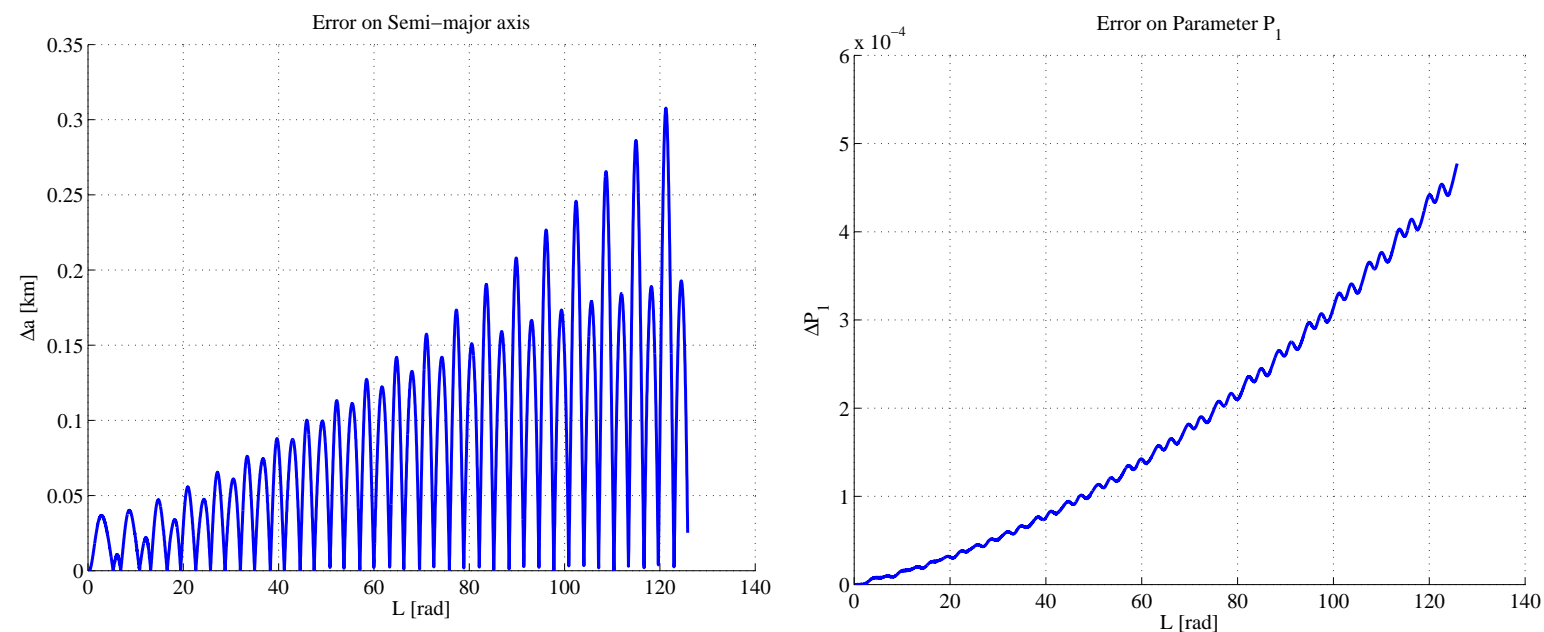

a)

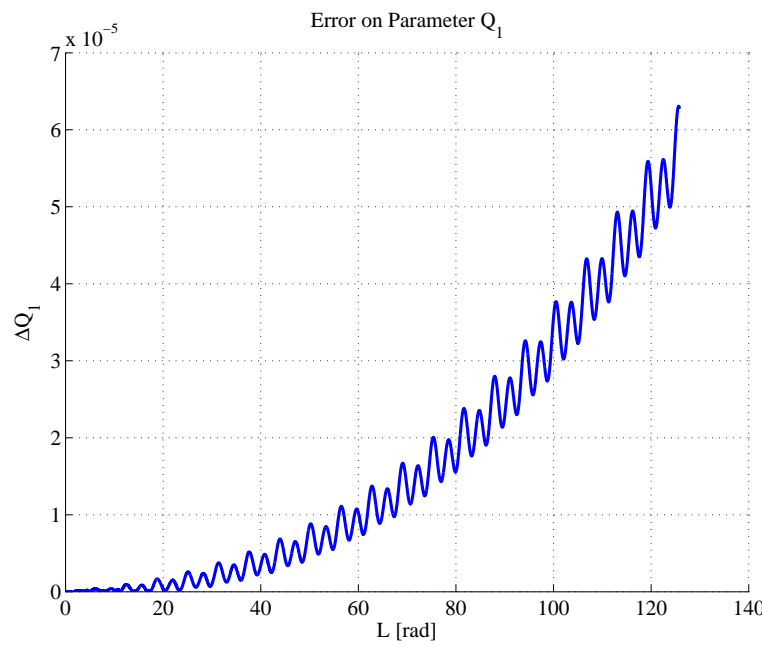

b)

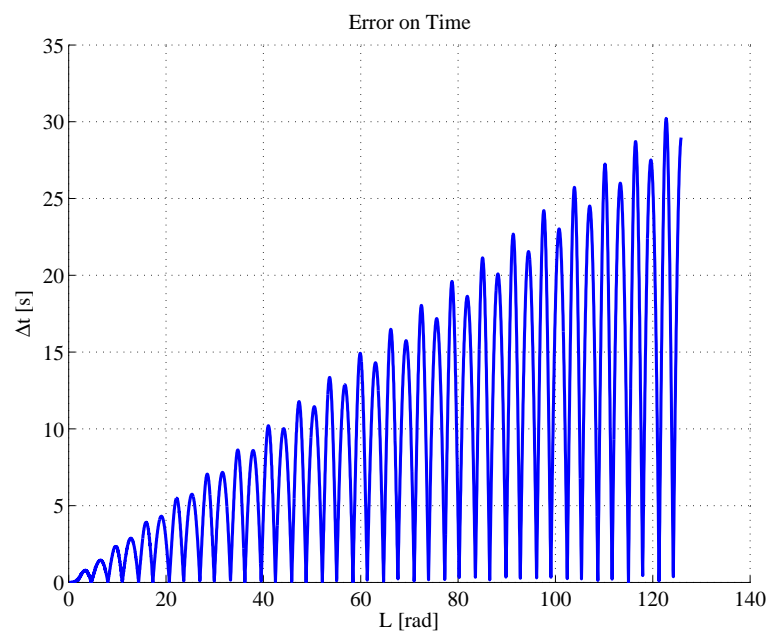

c)

d)

Fig. 7: Combined perturbations: a) error on semi-major axis. b) error on $P_{1}$. c) error on $Q_{1}$. d) error on time.

Fig. 7a shows that the error on the semi-major axis remains contained below $0.3 \mathrm{~km}$ after 20 revolutions. Fig. 7b and Fig. 7c show that the analytical approximation of $P_{1}$ and $Q_{1}$ is very close to the numerical solution with errors lower than $5 \times 10^{-4} . P_{2}$ and $Q_{2}$ show similar behaviours but are omitted here for the sake of conciseness.

Fig. 7d shows the difference on time of flight between the approximation computed with [37] and the numerical integration of the time equation. The approximated time of flight 
accumulates an error that is less than $30 \mathrm{~s}$ after 20 orbits (a relative error of $5 \times 10^{-3}$ ). This result demonstrates that the new approximation proposed in [16] reduces the error by one order of magnitude with respect to the previous formulation proposed in Zuiani et al. (2012) for the same ratio of control and local gravity acceleration. Note that a good computation of the elapsed time is essential, in particular when one has to use this datum to compute the $\Delta V$ corresponding to the propagated thrusting arc.

In this test the analytical propagation required about $1.5 \times 10^{-3} \mathrm{~s}$ compared to $0.8 \mathrm{~s}$ of the numerical integration with ode113. If the tolerance on the relative and absolute error of the numerical integration is relaxed to $10^{-5}$ (a relative error comparable to the one of the analytical solutions), the CPU time for ode113 reduces to about $0.24 \mathrm{~s}$, which is still two orders of magnitude slower than the analytical propagation.

\subsubsection{Propagation Error Control Over Long Spiralling Arcs}

The results in the previous sections show that the propagation error of the proposed analytical solutions remains contained over relatively long arcs provided that $\varepsilon$ is small compared to the local gravity field (see Zuiani et al. 2012a). For longer spirals, however, an error control strategy is required to preserve accuracy even for small values of $\varepsilon$.

The propagation error can be controlled by updating the reference condition $\mathbf{E}_{0}$ in [11] and [16] every $n$ orbits. The update consists of taking the value for $\mathbf{E}(L)$ computed at the $n$-th orbit as the new reference condition $\mathbf{E}_{0}$ for the following $n$ orbits. This technique, presented in Colombo et al. (2009), can be regarded as a rectification of the analytical propagation.

The effectiveness of the technique is demonstrated here with the propagation of an initial circular orbit with a semi-major axis of $7000 \mathrm{~km}$ under the effect of an acceleration $\varepsilon=10^{-4}$ $\mathrm{m} / \mathrm{s}^{2}$ along the tangential direction. The length of the propagation arc is equal to 500 complete revolutions. The frequency of updates is set to $n=20$, leading to a total of 25 evaluations of the analytical formulae. The analytical propagation required about $0.03 \mathrm{~s}$ while a numerical one with ode113 (Adams-Bashfort, with tolerance set to $10^{-13}$ ) took about 7 s. Fig. 8 a shows that the relative error in the semi-major axis remains below $5 \times 10^{-4}$. 


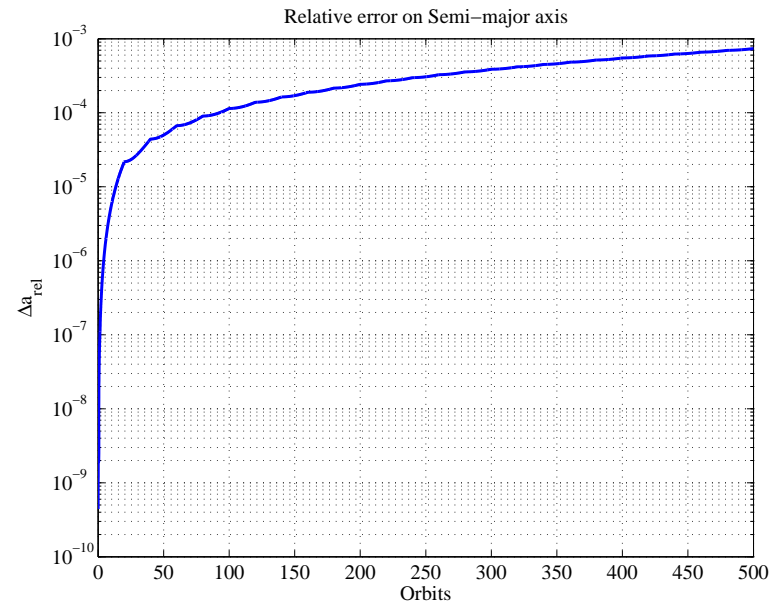

a)

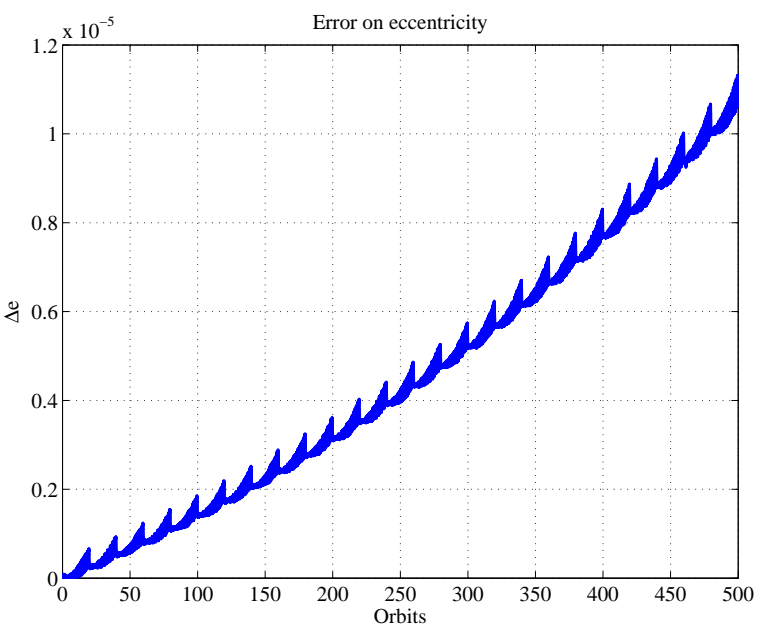

b)

Fig. 8: LEO propagation: a) relative error on semi-major axis. b) error on eccentricity.

Fig. $8 \mathrm{~b}$ shows the effect of the rectification process on the error in eccentricity, which remains below $1.2 \times 10^{-5}$ after 500 orbits.

A further test considers the propagation from an initial Geostationary Transfer Orbit (see Table 1) until escape condition is reached with an acceleration $\varepsilon=10^{-4} \mathrm{~m} / \mathrm{s}^{2}$ in the tangential direction. This time, due the fact that the transfer is much longer and will span a wide range of different orbit energies, a simple dynamic rule is implemented to define the frequency of rectification. As detailed in Table 3, the frequency of rectification is proportional to the value of the semi-major axis. The analytical propagation was compared to a numerical integration with ode113. The computational times are around $0.6 \mathrm{~s}$ for the analytical propagation and $15 \mathrm{~s}$ for ode113. Similar to the previous case, Fig. 9 show, respectively, the variation of semimajor axis, the eccentricity and the relative error on radius modulus and that on phasing. Fig. 9a shows a very good match between the analytical and numerical propagations, as can also be seen in Fig. 9b for the eccentricity. The match is very good up to the last few revolutions when the semi-major axis is very large and the analytical formulae become relatively inaccurate. Fig. 9d shows the relative error on the modulus of the position vector. The figures show that the relative error remains below $10^{-3}$ for a good part of the spiral and grows above $10^{-2}$ only towards the end when the semi-major axis grows above $5 \times 10^{4} \mathrm{~km}$. Fig. 9e shows the phasing error as the angular distance between the radius vector computed analytically and the one computed from the numerical integration. As can be seen this stays always below $10^{-2}$ rad.

\begin{tabular}{|c|c|c|c|c|}
\hline Interval $\left[10^{4} \mathrm{~km}\right]$ & $a<3$ & $3<a<8.5$ & $8.5<a<10$ & $a>10$ \\
\hline Rectifications/orbit & 1 & 2 & 4 & 8 \\
\hline
\end{tabular}




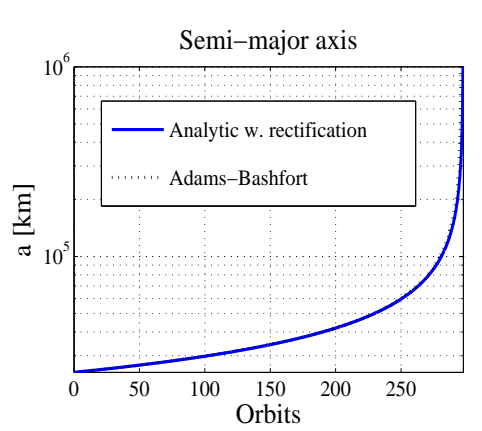

a)

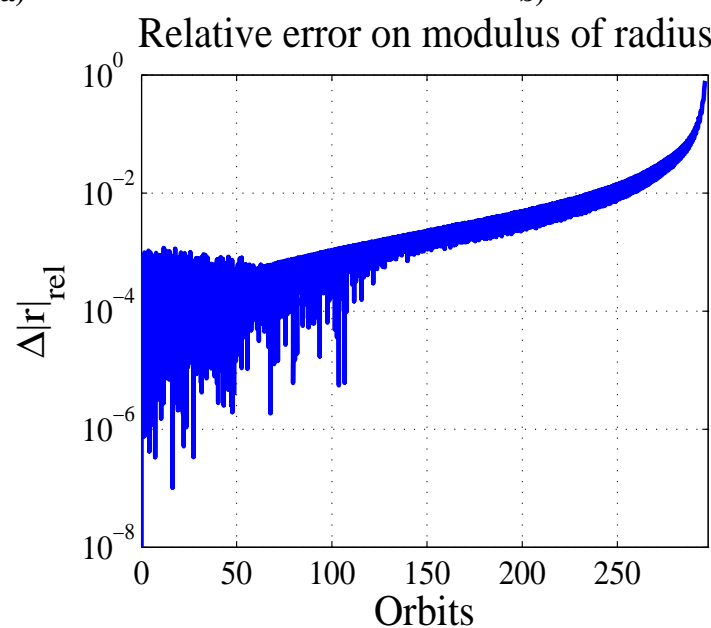

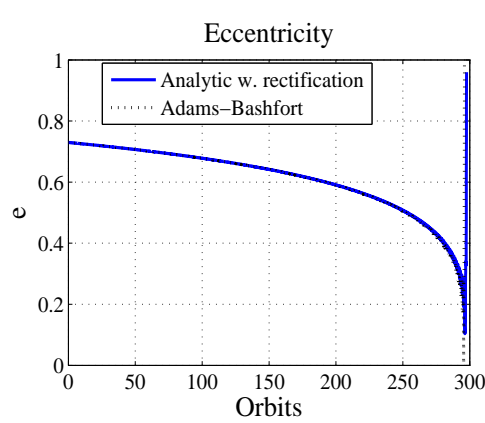

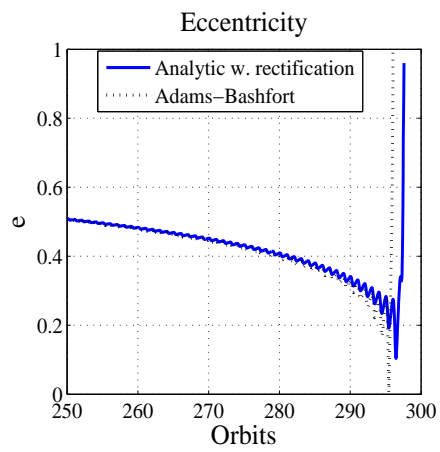

c)

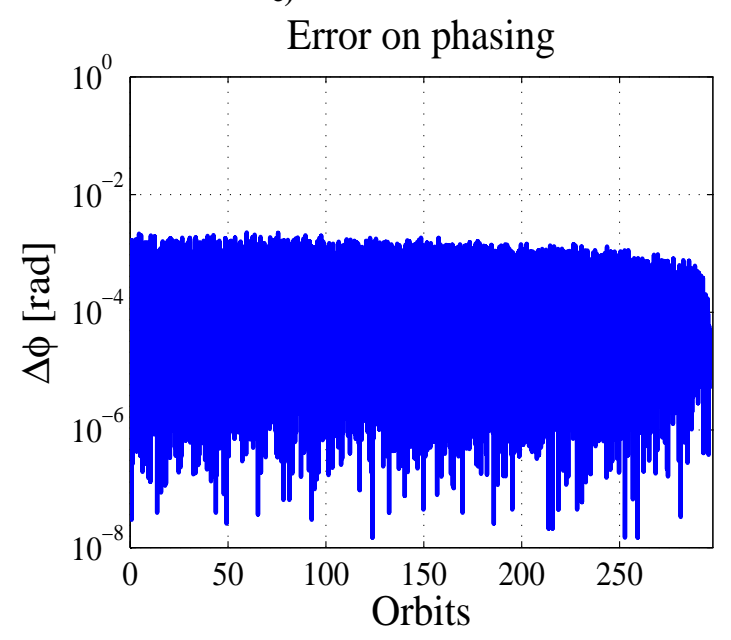

e)

d)

Fig. 9: GTO propagation: a) semi-major axis. b) eccentricity, whole trajectory. c) eccentricity, magnification of last orbits. d) relative error on radius. e) phasing error.

\section{Discontinuous Control Profiles and Eclipses}

In the numerical tests presented in the previous section, a simple continuous acceleration was considered. This section proposes a simple approach to introduce bang-zero-bang control profiles and eclipses. 


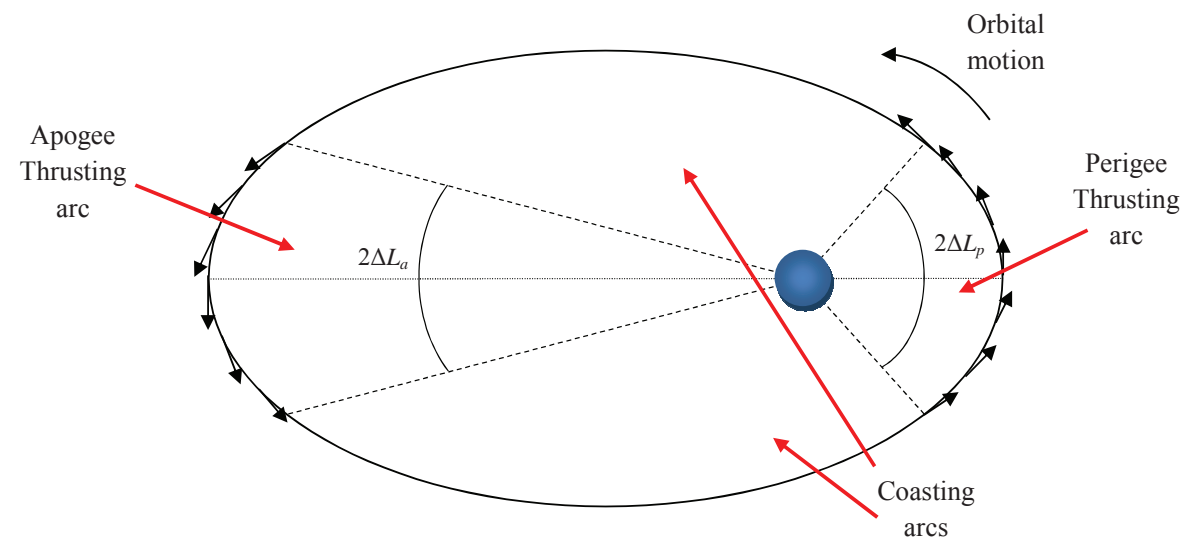

Fig. 10: Control pattern.

The interest is in the class of low-thrust transfers whose quasi-optimal control corresponds to two thrust arcs. For this class of transfers, each revolution can be divided into 4 sectors, as shown in Fig. 10: a perigee thrust arc, an apogee thrust arc and two coasting arcs in between. The former, of amplitude $\Delta L_{p}$, is meant to alter the radius of the apocentre, while the latter, of amplitude $\Delta L_{a}$, alters the radius of the pericentre. The combined effect of the two thrust arcs can be used to control the inclination and the argument of the pericentre. The variation of the orbital elements along the thrusting arcs is computed with the analytical formulae. A plane change is realised introducing a non-zero elevation angle $\beta_{p}$ and $\beta_{a}$. The amplitude of the arcs $\Delta L_{p}$ and $\Delta L_{a}$, and the angles $\beta_{p}$ and $\beta_{a}$, are the quantities to be controlled to match the desired terminal conditions. When a constant thrust is required for each arc, the mass of the spacecraft $m_{f}$ at the end of a thrust arc can be estimated assuming the control acceleration $\varepsilon$ is constant along the thrust arc:

$$
m_{f}=m_{i} e^{-\frac{\varepsilon \Delta t}{I_{s p} g_{0}}}
$$

where $m_{i}$ is the mass of the spacecraft at the beginning of the thrust arc. The new mass is then used to recompute the control acceleration for the next thrust arc.

\subsection{Eclipse Modelling}

In the case of long, multi-revolution transfers, the effect of a solar eclipse might be considerable if one takes into account the fact that, for example, during an eclipse the operation of an electric propulsion system will, most likely, have to be interrupted due to limitations on power generation and storage. Moreover, eclipses change due to the combined effect of the motion of the Earth around the Sun and the variation of orbit size and orientation due to engine thrust and other perturbative effects. In the case of a full numerical integration, 
eclipses are computed by checking shadow conditions at each step and eventually activating or deactivating some kind of eclipse flag. This leads to discontinuities in the integrand function. In an analytical approach, like the one here proposed, one can however exploit the fact that the entrance and exit points of the shadow cone (for the sake of simplicity, no distinction is made between umbra and penumbra conditions) can be computed beforehand and then the thrusting arcs can be updated accordingly. Other authors have already proposed a similar approach, see for example Kechichian (1998a), in which orbits with eccentricity up to 0.2 are considered; Colombo and McInnes (2011) also applied a similar method but limited to the planar case. In this work, a cylindrical model for Earth's shadow is adopted (see Fig. 11 ), which is perfectly adequate in the case of a spacecraft in Earth orbit.

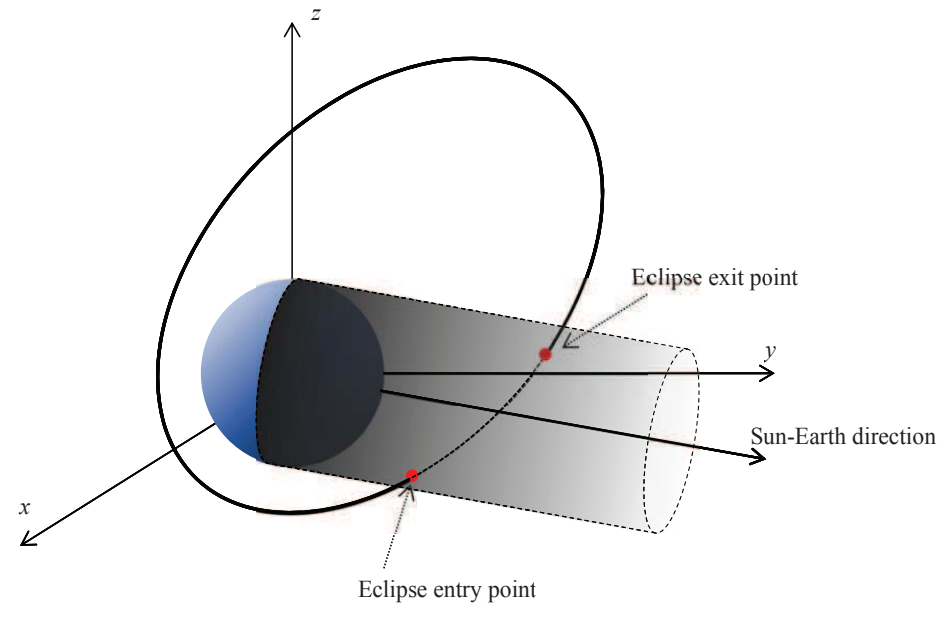

Fig. 11: Shadow model.

In order to identify the eclipse entry and exit points one has to find the true anomalies of the geometrical intersections between the cylinder and the osculating orbit. The mathematical formulation of this problem can be found in Escobal (1965) and Vallado (2007) and will not be repeated here. Starting from the osculating orbital elements and the current Sun-Earth vector, this formulation leads to a quartic equation in $\cos \theta$, which can be solved either analytically by means of Ferrari's method, as is done in this work, or numerically with a rootfinding algorithm. Note that out of the 4 roots of the quartic polynomial, two are spurious. Once the shadow entry and exit points are known, one can correct the thrusting and coasting arcs as shown in Fig. 12. 


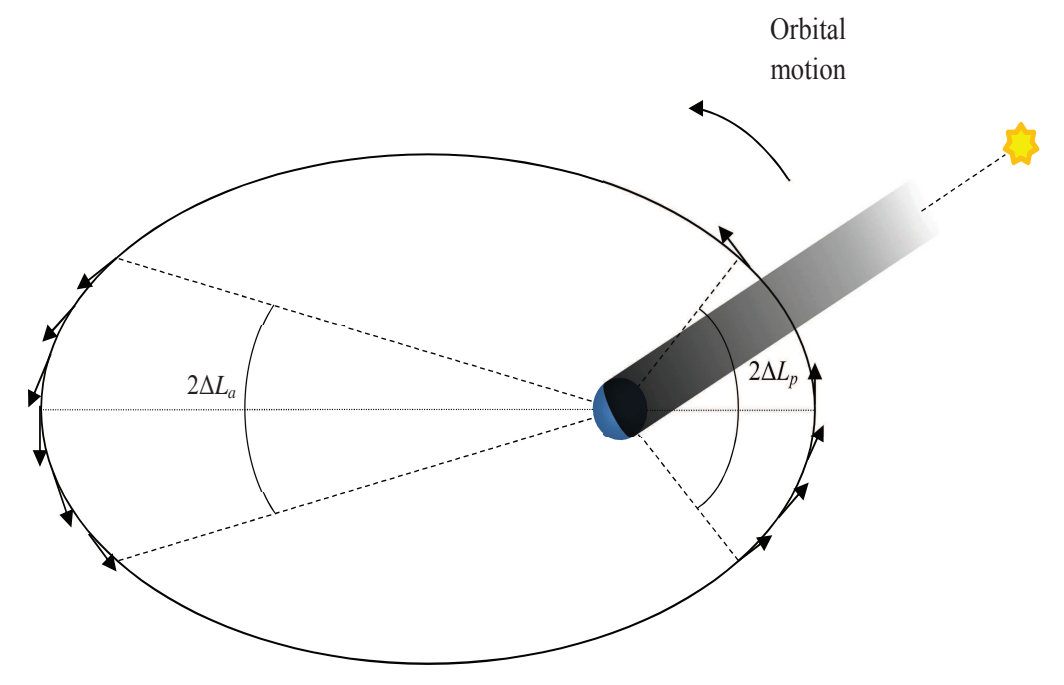

Fig. 12: Thrust pattern with eclipse region.

Apart from identifying the shadow regions, this formulation also allows one to analytically compute the time spent in the shadow region, $t_{e c l}$, for each orbit.

\section{Applications}

This section presents a number of applications of the analytical formulae to cases of practical interest. In particular, it will be shown how the analytical formulae can be effectively used to implement an orbit averaging technique.

\section{1. $\quad$ Orbit Averaging}

In the previous sections, a set of analytical formulae for propagating the perturbed Keplerian motion was proposed. As a further step, it is proposed to use these formulae to compute the average variation of the orbital elements over a complete revolution and to numerically propagate the average orbital elements, as it is done in classical averaging techniques (see Ferrier and Epenoy 2001, Tarzi et al. 2013). In the proposed averaging technique, the variation of the orbital elements is given by:

$$
\begin{aligned}
& \overline{\mathbf{E}}(t)=\mathbf{E}_{0}+\int_{t_{0}}^{t} \dot{\mathbf{E}}_{a v g}\left(\tau, \overline{\mathbf{E}}(\tau), \overline{\Delta L}_{p}(\tau), \overline{\Delta L}_{a}(\tau), \bar{\beta}_{p}(\tau), \bar{\beta}_{a}(\tau)\right) d \tau \\
& \dot{\mathbf{E}}_{\text {avg }}=\frac{\Delta \mathbf{E}_{2 \pi}}{T_{2 \pi}}
\end{aligned}
$$

where $\overline{\mathbf{E}}$ represents the vector of the averaged orbital elements. $\Delta \mathbf{E}_{2 \pi}$ is the net variation of the orbital elements computed over a complete revolution and $T_{2 \pi}$ is the corresponding 
period. In some orbit averaging techniques, $\Delta \mathbf{E}_{2 \pi}$ is computed by numerical quadrature of Gauss' planetary equations over the true anomaly (or longitude). Here $\Delta \mathbf{E}_{2 \pi}$ is provided by the evaluation of the analytical formulae at $L=2 \pi$. As shown in Section 3 , this is advantageous in terms of computational cost for a comparable accuracy. The terms $\overline{\Delta L}_{p}$, $\overline{\Delta L}_{a}, \bar{\beta}_{p}$ and $\bar{\beta}_{a}$ are the control parameters mentioned in Section 4 and are computed as a piecewise linear interpolation with respect to time, from $n_{\text {nodes }}$ nodal values, uniformly spaced within the limits of the transfer period. For example, in the case of $\overline{\Delta L}_{p}$, one can write:

$$
\overline{\Delta L}_{p}(t)=f_{\text {interp }}\left(\mathbf{t}_{p}, \Delta \mathbf{L}_{p}, t\right)
$$

where $\Delta \mathbf{L}_{\mathrm{p}}$ is a vector containing the $n_{\text {nodes }}$ nodal values, $\mathbf{t}_{\mathbf{p}}$ is the vector which collects the corresponding times at which the nodal values are specified, and $f_{\text {interp }}$ defines a piecewise linear interpolation. The tests in Section 3 have already shown that the analytical propagation is advantageous over numerical quadrature even in the case of discontinuous thrusting profiles over a single revolution.

\subsection{Spiralling with Solar Radiation Pressure and Eclipses}

This section presents a comparison between the averaged solution and the rectified solution for the case of a long term propagation of an initial planar elliptical orbit (as in Table 4) under the combined effect of a thrust acceleration along the tangential direction, solar radiation pressure (SRP) and Earth oblateness $\left(J_{2}\right.$ effect).

\begin{tabular}{|c|c|c|c|c|c|}
\hline$a$ & $E$ & $i$ & $\Omega$ & $\omega$ & $\theta$ \\
\hline $20000 \mathrm{~km}$ & 0.5 & $0^{\circ}$ & $0^{\circ}$ & $0^{\circ}$ & $0^{\circ}$ \\
\hline
\end{tabular}

Table 4: Initial orbit parameters.

The initial mass of the spacecraft is $1000 \mathrm{~kg}$, and it is assumed that the engine delivers $10^{-2} \mathrm{~N}$ at a specific impulse of $3000 \mathrm{~s}$. The cross section area used to compute the SRP acceleration is $1200 \mathrm{~m}^{2}$, a value chosen so that the resulting force is about half of the thrust of the engine. At departure, the Sun lies at the Summer Solstice point. The propagation time is set to one and a half years. The SRP direction is considered to be constant along an orbit, therefore allowing the use of the formulas in [23] for a constant inertial acceleration. The secular variation in the Sun-Earth direction is used to update the direction of the inertial acceleration. Moreover, eclipses are introduced with the methodology detailed in Section 4.1 and the consequent thrust interruptions are accounted for. The averaged propagation is performed with MATLAB ${ }^{\circledR}$ ode 23 which implements a Runge-Kutta integration method. The results are 
compared to a full numerical integration with ode113, and to the analytic propagation with rectification every orbit if no eclipse occurs or two times per orbit if an eclipse is present. The CPU time required by the averaged analytic propagation was $1.2 \mathrm{~s}$ while the full numerical integration required about $100 \mathrm{~s}$. The rectification required about $7 \mathrm{~s}$, showing the additional advantage of the analytic averaging approach. Fig. 13a shows the long-term, monotonic increase of the semi-major axis due to the tangential thrust. Fig. 13b shows a close up of a portion of the curve. The dashed curve represents the full numerical integration, the dotted curve represents the analytical propagation with rectification and the solid curve the average solution. Note that the analytical formulas are evaluated only at the end of each revolution and at the transition out of the eclipse. The full numerical integration displays a short-term oscillation of $a$, due to $J_{2}$ and SRP, a secular increment over a revolution. The averaged solution captures accurately the secular components while the analytical solution with rectification keeps track of the periodic components, although in the figure only the value of the semi-major axis at the eclipse times is plotted.
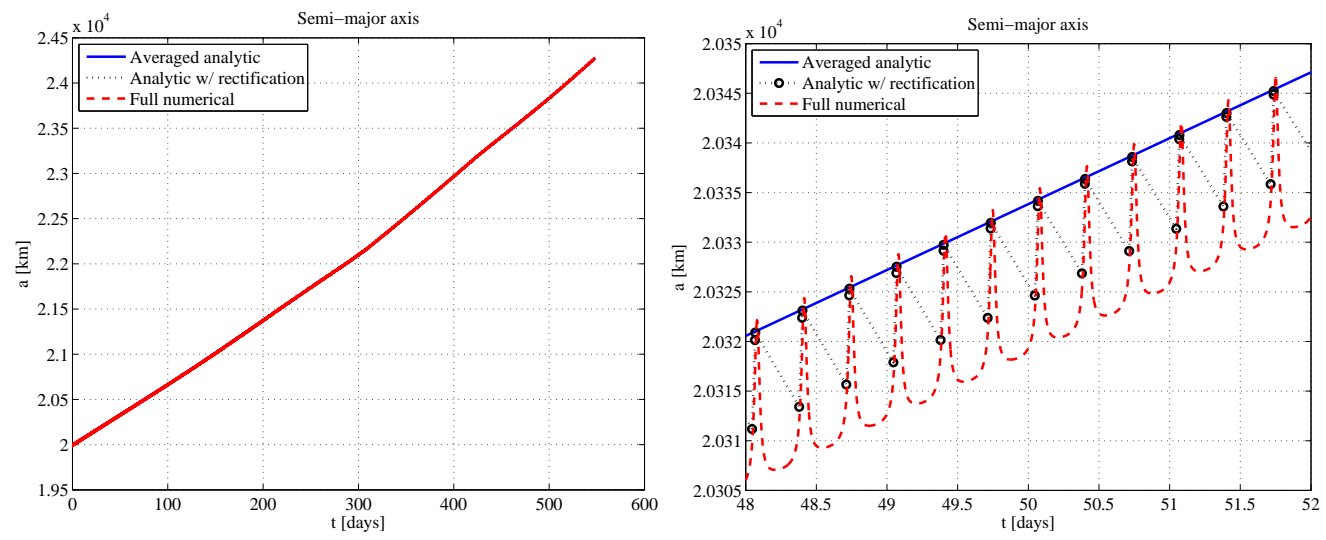

Fig. 13: Spiralling with SRP: a) semi-major axis; b) Close-up.

Fig. 14a shows the long term variation of orbital eccentricity due to the combined effects of tangential thrust and SRP. Tangential thrust alone would produce a monotonic decrease of the eccentricity, however, SRP adds a long-term oscillatory component that is linked to the rotation of the Sun-spacecraft vector. SRP also produces a small long term deviation of the inclination due to the relative angle between the Ecliptic plane and the Equatorial plane, in which the initial orbit lies, as shown in Fig. 14b. 

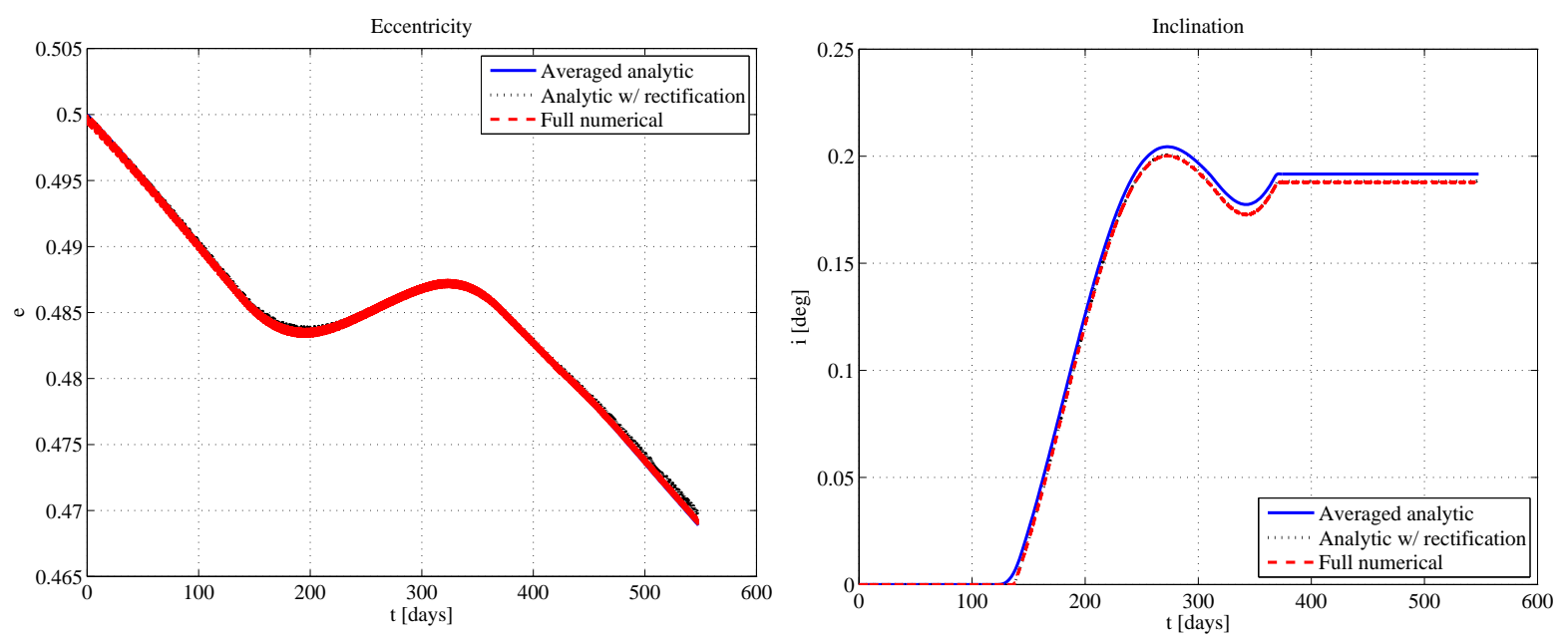

a)

b)

Fig. 14: Spiralling with SRP: a) eccentricity. b) inclination.

\subsection{GTO to GEO Orbit Circularisation}

The previous section demonstrates the advantage of using the proposed analytical formulae for the long propagation of spirals with discontinuous control profiles and eclipses together with their use in conjunction with orbit averaging. In this section a further example will demonstrate how to combine the control parameterisation presented in Section 4 with orbit averaging to circularise an initial GTO into a GEO in a specified transfer time. The initial orbit parameters are as in Table 5.

\begin{tabular}{|c|c|c|c|c|c|}
\hline$a$ & $e$ & $i$ & $\Omega$ & $\omega$ & $\Theta$ \\
\hline $24505.9 \mathrm{~km}$ & 0.725 & $7^{\circ}$ & $0^{\circ}$ & $0^{\circ}$ & $0^{\circ}$ \\
\hline
\end{tabular}

Table 5: Initial orbit parameters.

The target orbit is a GEO with zero inclination, therefore a plane change of $7^{\circ}$ is also required. The time specified for the transfer is 225 days. Engine thrust is $0.35 \mathrm{~N}$, with a specific impulse of $2000 \mathrm{~s}$. The initial mass of the spacecraft is $2000 \mathrm{~kg}$ and mass consumption is taken into consideration during the transfer using [38]. Four nodes each are used to model the variation of $\Delta L_{p}, \Delta L_{a}, \beta_{p}$ and $\beta_{a}$, leading to a total of 16 optimisation parameters. The total $\Delta V$ is minimised while matching the final semi-major axis, eccentricity and inclination, obtained through the analytical propagator, with those of the target orbit: 


$$
\begin{aligned}
& \min _{\Delta L_{p}, \Delta L_{a}, \beta_{p}, \beta_{a}} \Delta V \\
& \text { s.t. } \\
& \mathbf{E}\left(t_{0}\right)=\mathbf{E}_{\text {GTO }} \\
& \mathbf{E}\left(t_{f}\right)=\mathbf{E}_{G E O}
\end{aligned}
$$

This is basically a single-shooting, direct collocation method. The MATLAB ${ }^{\circledR}$ fmincon-sqp algorithm is used to solve problem [41]. The optimisation converges in 8 iterations and 25 seconds (on a Windows 7 platform), and the optimised solution has a $\Delta V$ cost of $1.78 \mathrm{~km} / \mathrm{s}$. This result compares well with that given, for an identical test problem, by the solver MIPELEC (see Ferrier and Epenoy 2001), which returns a $\Delta V$ cost of $1.68 \mathrm{~km} / \mathrm{s}$ in about $14 \mathrm{~s}$ of computational time on a UNIX-based Sun workstation. To compare the computational times, one has to consider that MIPELEC is written in FORTRAN77 and a MATLAB code is usually at least one order of magnitude slower than an equivalent FORTRAN code.
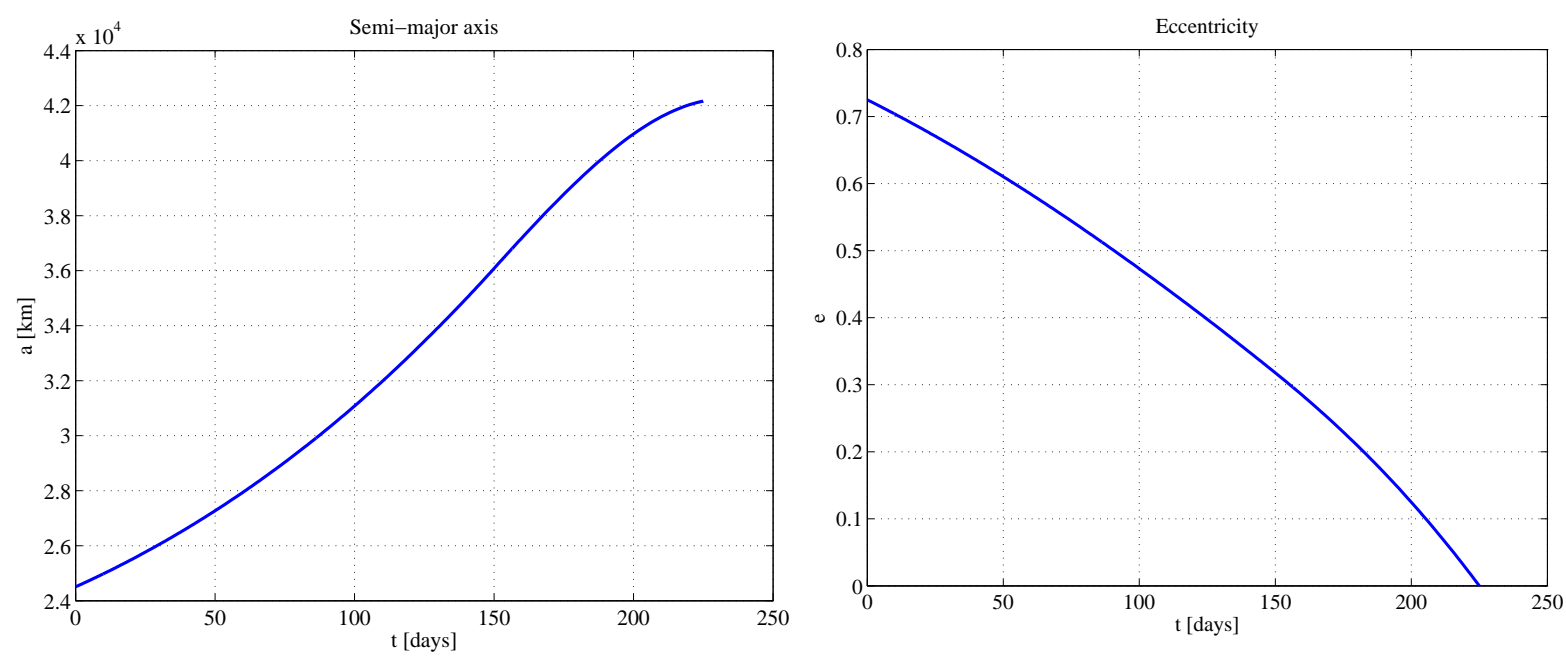

a)

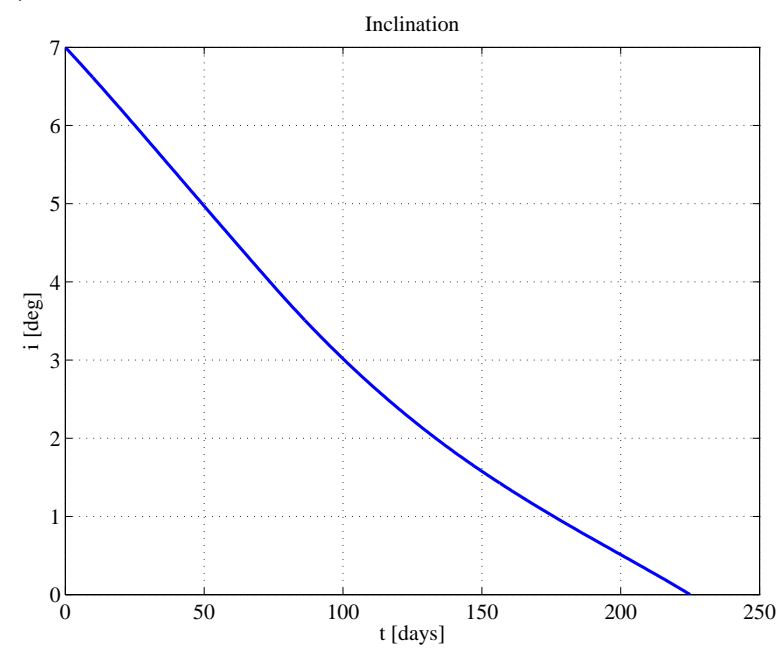

b)

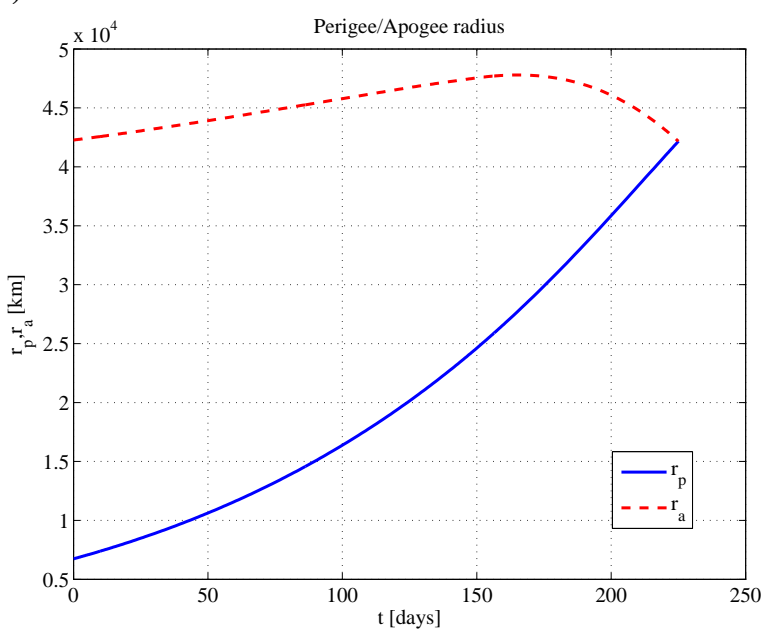

c)

d) 

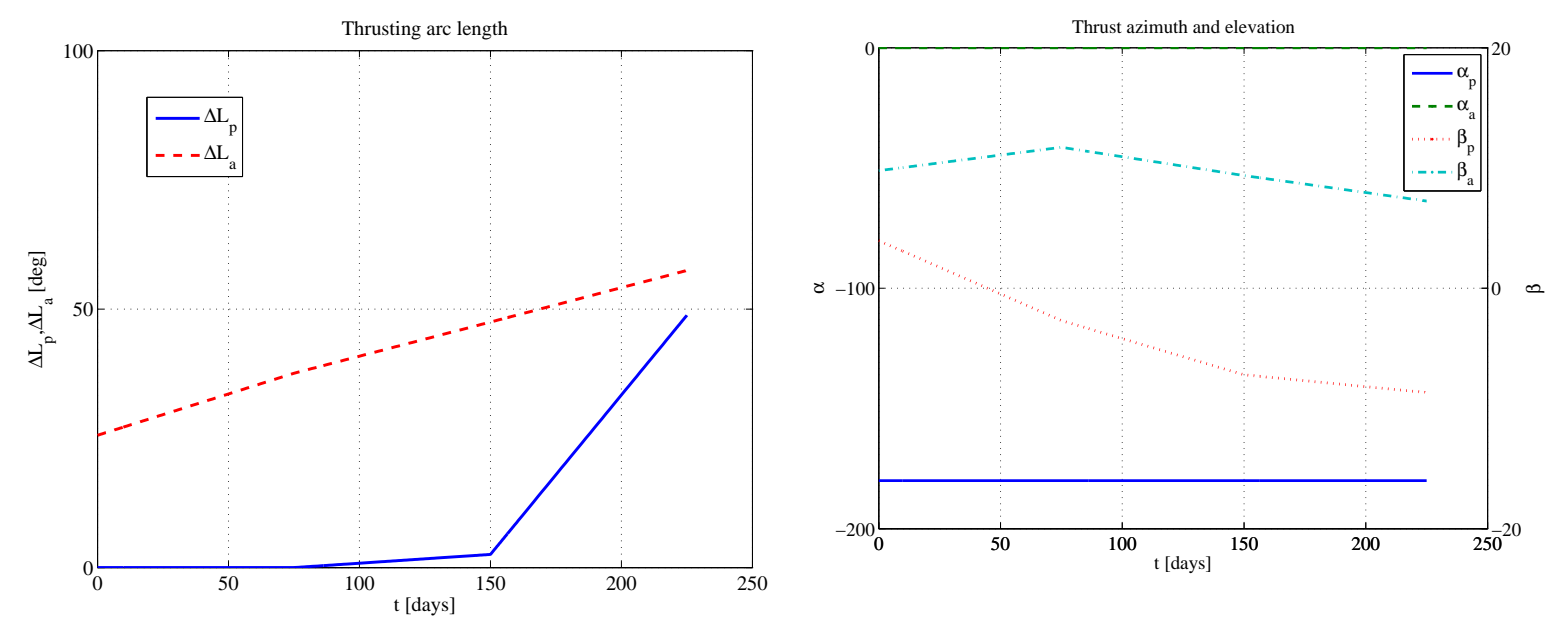

e)

f)

Fig. 15: Orbit circularisation: a) semi-major axis. b) inclination. c) perigee and apogee. d) perigee and apogee. e) thrusting arc length. f) thrust azimuth and elevation.

Fig. 15a, Fig. 15b and Fig. 15c show the variation of semi-major axis, eccentricity and inclination respectively. It can be seen that all quantities change monotonically from their initial values to the target ones.

Fig. 15d shows the variation of perigee and apogee and it is interesting to see that the perigee rise gradually increases in speed. There is also a slight increase in the apogee radius due to the amplitude of the apogee thrusting arc which is compensated for in the last part of the transfer by a perigee arc (see Fig. 15e), with thrust in the negative tangential direction. Note that these behaviours are consistent with the results shown in Geffroy and Epenoy (1997) and Tarzi et al. (2013), even if here a much more simplified model has been used.

Fig. 15f shows the thrust azimuth $\alpha$ and elevation $\beta$ in the $t-n$ - $h$ reference frame for the perigee and apogee thrusting arcs. It shows that the apogee arc always has a positive tangential component (i.e. energy-increasing), while the opposite is true for the perigee one since it has to compensate for the apogee altitude increase. The plane change effort is concentrated at the apocentre with an out-of-plane component around $15^{\circ}$. Note that the contribution of perigee thrusting to the plane change is only during the final part of the circularisation.

\section{Conclusions}

This paper has presented a set of analytical formulae for perturbed trajectories under the effect of a constant control acceleration, solar radiation pressure and $J_{2}$ effect. The proposed approach is suitable for treating constant accelerations in the $r-\theta-h, n-t-h$ and inertial reference frames. The accuracy of the analytical solutions was shown to be good for the 
propagation of relatively long trajectory arcs around the Earth, if the control acceleration level is comparable to the one delivered by current low-thrust engines.

A rectification technique was then proposed to control the propagation error and to accurately propagate long spiralling trajectories. Finally, by introducing a simplified parameterisation for the thrusting pattern, the proposed approach was applied to the optimal design of long spirals with terminal constraints in combination with orbit averaging.

The good accuracy displayed in the experimental tests and the fast propagation speed make the proposed analytical theory particularly suitable for the global multi-objective optimisation of low-thrust spirals.

\section{Acknowledgments}

The authors would like to thank Dr. Richard Epenoy from CNES for his helpful suggestions and for the results of the test case with MIPELEC.

\section{References}

Casalino, L., Colasurdo, G. Pastrone, D.: Optimal Low-Thrust Escape Trajectories Using Gravity Assist, Journal of Guidance, Control, and Dynamics, Vol. 22 No. 5 (1999)

Conway, B.A., Tang, S.: Optimization of low-thrust interplanetary trajectories using collocation and nonlinear programming, Journal of Guidance, Control, and Dynamics, Vol.18, No.3, 599-604 (1995)

Sims, J.A., Flanagan, S.N.: Preliminary Design of Low-Thrust Interplanetary Missions, Paper AAS 99-338 (1999)

Yam, C.H., Di Lorenzo, D., Izzo, D.: Constrained Global Optimization of Low-Thrust Interplanetary Trajectories, Proceedings of the Twelfth conference on Congress on Evolutionary Computation (CEC 2010) (2010)

Kechichian, J.A.: Reformulation of Edelbaum's low-thrust transfer problem using optimal control theory, Journal of Guidance, Control, and Dynamics, Vol. 20, No. 5, 988-994 (1997)

Kechichian, J.A.: The treatment of the earth oblateness effect in trajectory optimization in equinoctial coordinates, Acta Astronautica, Vol. 40, 69-82, Elsevier (1997)

Kechichian, J.A.: Low-Thrust Eccentricity-Constrained Orbit Raising, Journal of Spacecraft and Rockets, Vol. 35, No. 3, 327-335 (1998)

Kechichian, J.A.: Orbit Raising with Low-Thrust Tangential Acceleration in Presence of Earth Shadow, Journal of Spacecraft and Rockets, Vol. 35, No. 4, 516-525 (1998)

Kechichian, J.A.: Low-thrust inclination control in presence of Earth shadow, Journal of Spacecraft and Rockets, Vol. 35, No. 4, 526-532 (1998) 
Kechichian, J.A.: The streamlined and complete set of the nonsingular J2-perturbed dynamic and adjoint equations for trajectory optimization in terms of eccentric longitude, The Journal of the Astronautical Sciences, Vol. 55, No. 3, 325-348 (2007)

Casalino, L. and Colasurdo, G.: Improved Edelbaum's Approach to Optimize Low Earth/Geostationary Orbits Low-thrust Transfers, Journal of Guidance, Control, and Dynamics, Vol. 30, Nr. 5, 1504-1510 (2007)

Geffroy, S. and Epenoy, R.: Optimal low-thrust transfers with constraints, generalization of averaging techniques, Acta Astronautica, Vol. 41, Nr 3, 133-149 (1997)

Kluever, C.A. and Oleson, S.R.: Direct approach for computing near-optimal low-thrust earth-orbit transfers, Journal of Spacecraft and Rockets, Vol. 35, No. 4, 509-515, AIAA (1998)

Gao, Y. and Li, X.: Optimization of low-thrust many-revolution transfers and Lyapunov-based guidance, Acta Astronautica, Vol. 66, No. 1, 117-129, Elsevier (2010)

Evtushenko, Yu.,G.: Investigation of the motion in a central field under the action of a constant tangential acceleration (in Russian), Prikl. Mat. Mekh., Vol. 30, No. 3 (1966)

Beletsky, V.V.: Essays on the Motion of Celestial Bodies, Birkhäuser Verlag (1999)

Colombo, C., Vasile, M. and Radice, G.: Semi-analytical solution for the optimal low-thrust deflection of NearEarth Objects, Journal of Guidance, Control and Dynamics, Vol. 32, No. 3, 796-809, AIAA (2009)

Bombardelli, C., Baù, G. and Peláez, J., Asymptotic solution for the two-body problem with constant tangential thrust acceleration, Celestial Mechanics and Dynamical Astronomy, Vol. 110, No. 3, 1-18, Springer (2011)

Lantoine, G., Russell, Ryan P.: “The Stark Model: an exact, closed-form approach to low-thrust trajectory optimization”, 21st International Symposium on Space Flight Dynamics (2009)

Lantoine, G., Russell, Ryan P.: "Complete closed-form solutions of the Stark problem”, Celestial Mechanics and Dynamical Astronomy, Vol. 103, No. 4, 333-366 (2011)

Zuiani, F., Vasile, M., Palmas, A., Avanzini, G.: Direct Transcription of Low-Thrust Trajectories with Finite Trajectory Elements, 61th International Astronautical Congress of the International Astronautical Federation, Prague, Czech Republic, IAC-10-C1.7.5 (2010)

Zuiani, F., Vasile, M., Palmas, A. and Avanzini, G.: Direct transcription of low-thrust trajectories with finite trajectory elements, Acta Astronautica, Vol. 72, 108-120, Elsevier (2012)

Zuiani, F., Vasile, M. and Gibbings, A.: Evidence-based robust design of deflection actions for near Earth objects, Celestial Mechanics and Dynamical Astronomy, Vol. 114, No. 1-2, 107-136 (2012)

Zuiani, F. and Vasile, M.: Preliminary design of Debris removal missions by means of simplified models for Low-Thrust, many-revolution transfers, International Journal of Aerospace Engineering, Hindawi (2012).

Zuiani, F., Kawakatsu, Y. and Vasile, M.: Multi-objective optimisation of many-revolution, low-thrust orbit raising for Destiny mission, 23rd AAS/AIAA Space Flight Mechanics Conference, Kauai, Hawaii, United States (2013)

Schoenmaekers J., Horas D., Pulido J.A.: SMART-1: With Solar Electric Propulsion to the Moon, International Symposium on Space Flight Dynamics, Pasadena, CA, USA, December (2001)

Russell, C.T., Capaccioni, F., Coradini, A., De Sanctis, M.C., Feldman, W.C., Jaumann, R., Keller, H.U., McCord, T.B., McFadden, L.A., Mottola, S., S., Pieters, C.M., Prettyman, T.H., Raymond, C.A., Sykes, M.V., Smith, D.E. and Zuber, M.T.: Dawn mission to Vesta and Ceres, Earth Moon Planets, Vol. 101, No. $1-2,65-91(2007)$ 
Smith, J.C., Parcher, D.W., and Whiffen, G.J.: Spiraling away from Vesta: design of the transfer from the low to high altitude Dawn mapping orbits, 23rd AAS/AIAA Space Flight Mechanics Conference, Kauai, Hawaii, United States (2013)

Battin, R.H.: An introduction to the mathematics and methods of astrodynamics, AIAA Education Series (1987)

Holmes, M. H.: Introduction to the foundations of applied mathematics, Springer (2009)

Sanders, J.J.A., Verhulst, F., Murdock, J.A.: Averaging methods in nonlinear dynamical systems, Springer (2007)

Carlson, B.C.: Computing elliptic integrals by duplication, Numerische Mathematik, Vol. 33, No. 1, 1-16 (1979)

Prince, P.J., Dormand, .J.R.: High order embedded Runge-Kutta formulae, Journal of Computational and Applied Mathematics, Vol. 7, No. 1, 67-75, Elsevier (1981)Colombo, C. and McInnes, C.: Orbital Dynamics of "Smart-Dust" Devices with Solar Radiation Pressure and Drag, Journal of Guidance, Control and Dynamics, Vol. 34, No. 6, 1613-1631 (2011)

Escobal, P.: Methods of Orbit Determination, New York, John Wiley and Sons (1965)

Vallado, D.A.: Fundamentals of Astrodynamics and Applications, $3^{\text {rd }}$ edition, Space Technology Library, Springer (2007)

Ferrier, C., Epenoy, R.: Optimal control for engines with electro-ionic propulsion under constraint of eclipse, Acta Astronautica, Vol. 48, No. 4, 181-192 (2001)

Tarzi, Z, Speyer, J., Wirz, R.: Fuel optimum low-thrust elliptic transfer using numerical averaging, Acta Astronautica, Vol. 86, 95-118 (2013) 